\title{
Biological Functions of Gasdermins in Cancer: From Molecular Mechanisms to Therapeutic Potential
}

\author{
Man Wang*, Xinzhe Chen and Yuan Zhang \\ Institute for Translational Medicine, The Affiliated Hospital of Qingdao University, Qingdao, China
}

Pyroptosis is a type of lytic programmed cell death triggered by various inflammasomes that sense danger signals. Pyroptosis has recently attracted great attention owing to its contributory role in cancer. Pyroptosis plays an important role in cancer progression by inducing cancer cell death or eliciting anticancer immunity. The participation of gasdermins (GSDMs) in pyroptosis is a noteworthy recent discovery. GSDMs have emerged as a group of pore-forming proteins that serve important roles in innate immunity and are composed of GSDMA-E and Pejvakin (PJVK) in human. The $\mathrm{N}$-terminal domains of GSDMs, expect PJVK, can form pores on the cell membrane and

OPEN ACCESS

Edited by:

Jochen H. M. Prehn,

Royal College of Surgeons in Ireland,

Ireland

Reviewed by:

Conor Henry,

Francis Crick Institute,

United Kingdom

Melissa Gillbert-Ross,

Emory University School of Medicine,

United States

*Correspondence:

Man Wang

wangman@qdu.edu.cn

Specialty section:

This article was submitted to

Cell Death and Survival,

a section of the journal

Frontiers in Cell and Developmental

Biology

Received: 07 December 2020

Accepted: 20 January 2021

Published: 09 February 2021

Citation:

Wang $M$, Chen $X$ and Zhang $Y$

(2021) Biological Functions

of Gasdermins in Cancer: From

Molecular Mechanisms

to Therapeutic Potential.

Front. Cell Dev. Biol. 9:638710. doi: 10.3389/fcell.2021.638710 function as effector proteins of pyroptosis. Remarkably, it has been found that GSDMs are abnormally expressed in several forms of cancers. Moreover, GSDMs are involved in cancer cell growth, invasion, metastasis and chemoresistance. Additionally, increasing evidence has indicated an association between GSDMs and clinicopathological features in cancer patients. These findings suggest the feasibility of using GSDMs as prospective biomarkers for cancer diagnosis, therapeutic intervention and prognosis. Here, we review the progress in unveiling the characteristics and biological functions of GSDMs. We also focus on the implication and molecular mechanisms of GSDMs in cancer pathogenesis. Investigating the relationship between GSDMs and cancer biology could assist us to explore new therapeutic avenues for cancer prevention and treatment.

Keywords: pyroptosis, cancer progression, gasdermins, prospective biomarkers, therapeutic avenues

\section{INTRODUCTION}

Gasdermins (GSDMs) are a conserved family of functionally diverse proteins which are mainly expressed in the gastrointestinal (GI) tract, skin and immune cells (Xia et al., 2019). In human, the GSDM family includes six members, GSDMA-E and PJVK (or autosomal recessive deafness type 59, DFNB59) (Van Laer et al., 1998; Delmaghani et al., 2006; Tamura et al., 2007). The GSDMA gene locates on chromosome 17 (17q21.1), and the GSDMB gene (17q12) is chromosomally located near GSDMA (Das et al., 2017). The GSDMC and GSDMD genes are located at 8q24 on human chromosome (Katoh and Katoh, 2004a; Saeki et al., 2009). GSDME, also named as deafness autosomal dominant 5 (DFNA5), is mapped at $7 \mathrm{p} 15$, while PJVK is located at $2 \mathrm{q} 31$ on human chromosome (Van Laer et al., 1997; Ebermann et al., 2007). In mice, ten GSDMs are discovered, including three homologs of GSDMA (GSDMA1-3), four homologs of GSDMC (GSDMC1-4), GSDMD-E and PJVK. So far, the biological functions have been elucidated for GSDMs. Each member of the GSDM family exhibits tissue- and developmental stage-specific expression patterns (Saeki et al., 2009). Accordingly, GSDMs perform regulatory function in a diversity of fundamental cellular processes, such as inflammation, cell proliferation, differentiation 
and death (Wang et al., 2013; Burgener et al., 2019). Remarkably, emerging evidence has indicated that GSDMs are associated with various human diseases, including cancer and inflammationdriven disorders (Hergueta-Redondo et al., 2014; Chao et al., 2017; Xu et al., 2018; Zhang et al., 2020). The N-terminal domains of GSDMs, except PJVK, have perforating capabilities that are blocked by their C-terminal fragments (Shi J. et al., 2015; Ding et al., 2016).

Importantly, GSDMs have been verified as critical molecules of the pyroptosis program (Wang et al., 2017, 2018b; Panganiban et al., 2018). Pyroptosis is a proinflammatory type of programmed cell death that frequently occurs upon microbial infection or other stimuli (Chang et al., 2020). Pyroptosis serves a vital role in the clearance of pathogenic infection and removal of endogenous danger signals by destroying the protective niche for pathogens and inducing immune responses (Kayagaki et al., 2011; Case et al., 2013). Inflammasome activation is considered as an original step during pyroptosis (Wu et al., 2010). Inflammasomes are innate immune sensors that monitor the cytosol for contamination or cellular perturbation, and in response they initiate pyroptosis (Fernandes-Alnemri et al., 2009; Thomas et al., 2009). Caspase1 activated by inflammasomes is required for converting the precursors of interleukin-1 $\beta$ (IL-1 $\beta$ ) and interleukin-18 (IL-18) into mature forms (Miao et al., 2010a). Meanwhile, GSDMs are cleaved into two parts mainly by inflammatory caspases, generating the $\mathrm{N}$-terminal and C-terminal fragments (Miao et al., 2010a). The N-terminal fragment (GSDM-NT) then couples with the inner leaflet of the cell membrane and oligomerizes into large pores. The generation of GSDM-NT pore dissipates cellular ionic gradients, leading to water influx, cell swelling and osmotic lysis with the extravasation of intracellular contents (He et al., 2015). In recent years, pyroptosis has become a new hotspot in cancer research. A growing body of evidence indicates that pyroptosis can suppress the proliferation and malignancy of cancer cells via induction of inflammatory cell death (Johnson et al., 2018; Tang et al., 2020). An in-depth investigation of the linkage between pyroptosis and cancers will provide new directions and molecular targets for the detection, treatment and prevention of cancers. Current research has centered on the molecules partaking in pyroptosis and molecular mechanisms underpinning pyroptosis. GSDMs, the key mediators of pyroptosis, have thus drawn increasing attention. GSDMs can act as oncoproteins/tumor suppressors and regulate cancer cell proliferation, invasion and metastasis. In addition, GSDMs are capable of fine tuning anticancer immunity. Taken together, GSDMs may represent attractive drug targets for effective cancer treatment. In this review, we provide an overview of the latest advances in the biological characteristics of GSDMs, with a focus on their roles and mechanisms involved in cancer biology.

\section{THE BIOLOGICAL CHARACTERISTICS OF GASDERMINS}

Besides PJVK, other GSDM members share approximately 45\% sequence homology and exhibit a similar architecture harboring an N-terminal functional domain and a C-terminal repressor domain (Lin et al., 2015; Chen Q. et al., 2019; Demarco et al., 2020). The two domains are connected by a linker containing a specific cleavage site for caspases. The C-terminal domain acts as an intrinsic repressor of GSDMs and can be removed via proteolytic cleavage, leading to the release of the pore-forming $\mathrm{N}$-terminal domain.

\section{Molecular Mechanisms of Pyroptosis}

GSDMA-E are capable of inducing pyroptosis once cleavage between their $\mathrm{N}$ - and C-terminal domains by inflammatory caspases (Figure 1). Pyroptosis is an inflammatory form of programmed cell death induced by caspases which are activated by specific inflammasomes. Pyroptosis was initially regarded as lysis taking place after the invasion of Shigella Flexneri in macrophages in 1992, which was erroneously categorized as apoptosis since earlier recognition of programmed cell death was restricted to apoptosis (Zychlinsky et al., 1992). Until 2001, Cookson and Brennan (Cookson and Brennan, 2001) first proposed the term "pyroptosis" to describe the caspase-1dependent programmed cell death that occurred in Salmonellainfected macrophages. Since the discovery of GSDMD, pyroptosis was redefined as GSDM-mediated programmed necrosis ( $\mathrm{He}$ et al., 2015; Kayagaki et al., 2015; Shi J. et al., 2015). Pyroptosis is featured by nuclear condensation, pore formation in the cell membrane, cell swelling with big bubbles, osmotic lysis and cell content leakage (Chen et al., 2016). Unlike apoptotic cells, cells undergoing pyroptosis maintain an intact nucleus (Bergsbaken and Cookson, 2007). The molecules implicating in pyroptosis have been discovered. Following activation of the canonical or non-canonical inflammasome pathways, pyroptosis can be impelled by inflammatory caspases (Chen et al., 2018; Yang et al., 2018). This discriminates pyroptosis from other forms of programmed cell death, such as apoptosis and necroptosis, which do not require inflammatory caspases. Inflammatory caspase-dependent pore formation on the cell membrane causes the disruption of cell membrane, resulting in the release of intracellular contents from the cell, including proinflammatory cytokines, alarmins and endogenous damage-associated molecular patterns (DAMPs) (Fink and Cookson, 2006).

Inflammasomes are multimeric complexes and generally consist of three components: a pattern-recognition receptor (PRR), an apoptosis-associated speck-like protein containing a caspase recruitment domain (ASC) and a caspase protease (Man et al., 2014). The PRR is responsible for detecting DAMPs induced by endogenous pathogens or pathogen-associated molecular patterns (PAMPs) derived from invading pathogens (Poltorak et al., 1998; Yang et al., 2010). The PRR family includes several members, such as Toll-like receptor (TLR), nucleotide-binding domain and leucine-rich repeat-containing receptor (NLR) and absent in melanoma 2 (AIM2)-like receptor (ALR) (Harton et al., 2002; Heil et al., 2004; Fernandes-Alnemri et al., 2009). The inflammasome-forming PRRs, NLRs and ALRs carry a caspase recruitment domain (CARD) or a pyrin domain (PYD) (Ting et al., 2008; Lu et al., 2015). The NLR family forms the principal group of inflammasome sensors. NLR proteins mainly include an N-terminal CARD or PYD, 


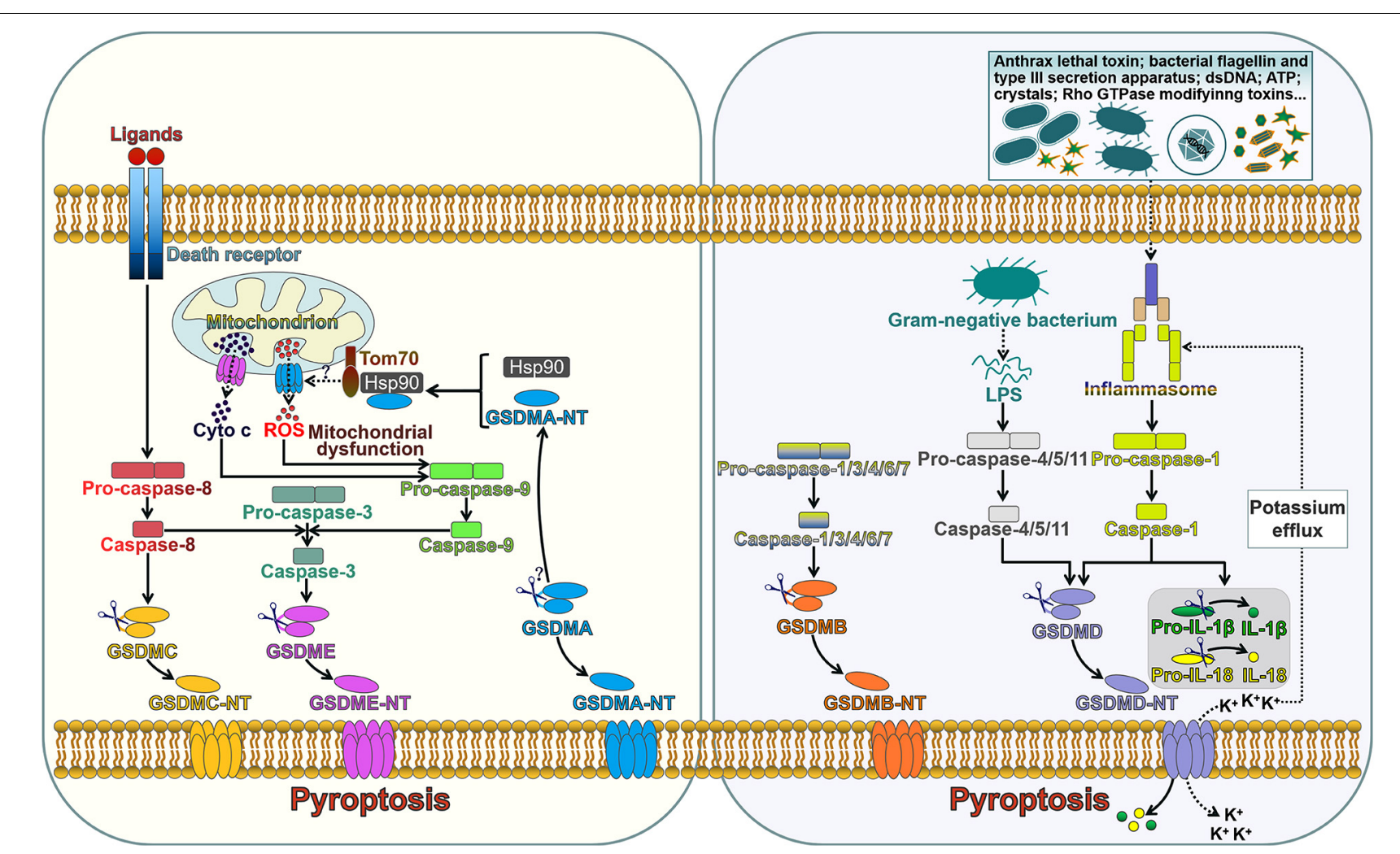

FIGURE 1 | The role of gasdermins in pore formation and pyroptotic cell death. The extrinsic pathway of apoptosis is triggered by interaction between death ligands and death receptors. The death receptor-mediated process involves the assembly of the death-inducing signaling complex (DISC) that induces the activation of caspase-8. GSDMC is cleaved into two fragments by caspase-8, generating an $\mathrm{N}$-terminal functional domain and a C-terminal repressor domain. The released GSDMC-NT exerts its pore-forming effect. Caspase-8 can cleave caspase-3. Active caspase-3 proteolytically processes GSDME, producing GSDME-NT to form pores on the cell membrane and induce pyroptosis. GSDME-NT is able to permeabilize the mitochondrial membrane that leads to the leakage of cyto $c$. The translocation of cyto $c$ to the cytosol can induce the activation of caspase- 9 that processes pro-caspase- 3 into active caspase-3. These events ultimately augment the apoptotic signaling cascade. GSDMA3-NT binds membrane lipids to form pores and triggers pyroptotic cell death. GSDMA3-NT combines with the cytosolic chaperone Hsp90 for mitochondrial targeting. Hsp90 escorts GSDMA3-NT to the mitochondrial transport receptor Tom70, leading to the generation of ROS. Mitochondrial dysfunction can result in the activation of caspase-9, which then processes pro-caspase-3 into caspase-3. GSDMB can be cleaved by caspase-1, -3 , $-4,-6$, and -7 . The unmasked $\mathrm{N}$-terminal domain punches massive holes in the cell membrane. The canonical inflammasome sensors recognize various DAMPs and PAMPs and activate caspase-1. Upon activation, caspase-1 cleaves GSDMD, releasing the N-terminal effector domain from the C-terminal inhibitory domain. GSDMD-NT interacts with phosphoinositides residing on the inner leaflet of the cell membrane and oligomerizes to generate membrane pores. This event leads to cell lysis and the release of intracellular contents. Caspase- 1 also cleaves the inactive proforms of IL-1 $\beta$ and IL-18 to generate biologically active cytokines. GSDMD-NT pores serve as a conduit for the passage of mature IL-1 $\beta$ and IL-18. Alternatively, caspase- $4,-5$, and -11 are activated by sensing intracellular LPS. Active caspase-4, -5 , and -11 cleave GSDMD within the linker domain. GSDMD-NT pores mediate potassium efflux, which in turn activates the canonical inflammasome signaling. Tom70, translocase of mitochondrial outer membrane 70; Hsp90, heat shock protein 90; cyto c, cytochrome c; ROS, reactive oxygen species; dsDNA, double-stranded DNA; ATP, adenosine triphosphate; LPS, lipopolysaccharide; GSDMA-E, gasdermin A-E; GSDMA-E-N T, the N-terminal domain of GSDMA-E; IL-1 $\beta$, interleukin-1 $\beta ;$ IL-18, interleukin-18.

a middle nucleotide-binding oligomerization domain (NOD), and C-terminal leucine-rich repeats (LRRs). The CARD/PYD is responsible for homotypic protein-protein interactions. NOD is required for adenosine triphosphate (ATP)-dependent activation of the signaling complex, while the LRR involves in ligand recognition and auto-inhibition. Upon activation, PRRs initiate the generation of a supramolecular assembly of ASC, which leads to the formation of ASC specks (Fernandes-Alnemri et al., 2007). CARD-carrying PRRs directly combine with pro-caspase1 through CARD-CARD interactions (Ball et al., 2020). In CARD-containing PRR-formed inflammasomes, caspase-1 can be activated via dimerization-induced auto-proteolysis. PYDcarrying PRRs cannot directly recruit pro-caspase- 1 . ASC, which is composed of an N-terminal PYD and a C-terminal CARD, acts as a scaffold protein associating PYD-containing PRRs with pro-caspase-1 (Proell et al., 2013). ASC is pivotal for caspase-1 activation via proximity-induced autoprocessing of pro-caspase1 (Guey et al., 2014). Recent findings have shown that caspase$1,3,4,5,8$ and 11 mediate the activation of inflammasome substrates (Shi J. et al., 2015; Wang et al., 2017; Orning et al., 2018). Pyroptosis can be driven mainly via two pathways, the canonical and non-canonical inflammasome pathways (Ruhl and Broz, 2015; Dubois et al., 2019).

\section{The Canonical Inflammasome-Induced Pyroptosis}

Canonical inflammasome is a cytosolic platform that activates caspase-1 after recognizing DAMPs or PAMPs (Wu et al., 2019). Generally, inflammasomes are multiprotein complexes 
that typically comprise a PRR (NLR or ALR), ASC and caspase1 (Guey et al., 2014). Five sensor proteins have been found to assemble canonical inflammasomes, and these include NLR family pyrin domain-containing 1 (NLRP1), NLRP3, NLR family CARD domain-containing 4 (NLRC4), AIM2 and pyrin (Newman et al., 2009; Wu et al., 2010; Xu et al., 2014). NLRP1, NLRP3 and NLRC4 belong to the NLR family, while AIM2 is a member of the ALR family (Kim et al., 2010; Rathinam et al., 2010). NLRP1 consists of an N-terminal PYD, a NOD, an LRR region, a function-to-find domain (FIIND), and a C-terminal CARD (Ting et al., 2008). NLRP1 directly binds to ASC via its PYD and recruits pro-caspase-1 via its CARD. NLRP1 responds to anthrax lethal toxin and muramyl dipeptide (Kovarova et al., 2012). NLRP3, one of the most studied inflammasome sensors, has an N-terminal PYD and NLR-typical elements (NOD and LRR) (Stutz et al., 2017). NLRP3 is able to recognize a variety of agonists, such as ATP, crystalline compounds, hyaluronan, nucleic acids, pore-forming toxins, as well as viral, bacterial and fungal pathogens (Mariathasan et al., 2006; Misawa et al., 2013; Hari et al., 2014). NLRC4 is composed of an N-terminal CARD domain, a central NBD domain, two hinge domains (HDs), a winged helix domain (WHD) and a C-terminal LRR domain (Moghaddas et al., 2018). NLRC4 is activated by bacterial flagellin and type III secretion apparatus in the cytosol (Miao et al., 2006, 2010b). AIM2, a ALR family member, is comprised of a DNA-binding HIN-200 domain and a PYD-signaling domain (FernandesAlnemri et al., 2009). AIM2 senses cytosolic double-stranded DNAs (dsDNAs) during pathogen invasion (Hornung et al., 2009). Pyrin is a unique inflammasome receptor protein that does not fit into any of currently known PRR families (Xu et al., 2014). The pyrin receptor holds a PYD domain, two B-boxes, a coiled-coil domain and a C-terminal SPRY/PRY domain (Yu et al., 2007). Pyrin mainly detects inactivating modifications of host Rho GTPases by various bacterial toxins or effectors ( $\mathrm{Xu}$ et al., 2014). The signaling domains of NLRs, AIM2 and pyrin combine with ASC by homotypic interactions, resulting in the formation of the ASC foci that subsequently recruit pro-caspase-1 (Broz et al., 2010; Proell et al., 2013). This eventually results in the activation of caspase- 1 . Specifically, NLRC4 directly recruits pro-caspase-1 and induces its activation (Li et al., 2018). Active caspase-1 not only mediates proteolytic cleavage of inactive IL-1 $\beta$ and IL-18 precursors, but also processes GSDMD to unleash its N-terminal poreforming domain (GSDMD-NT) (Miao et al., 2010a). GSDMDNT punches various holes in the cell membrane, facilitating the release of IL-1 $\beta$ and IL-18 into extracellular space and initiating inflammatory responses. The activation of caspase-1 also results in DNA fragmentation (Fink and Cookson, 2006). The canonical inflammasome-induced pyroptosis serves as a defense mechanism against pathogen infection and forms a critical portion of the innate immune system.

\section{The Non-canonical Inflammasome-Induced Pyroptosis}

In the non-canonical inflammasome-induced pyroptosis pathway, human caspase-4/-5 or murine caspase-11 can be directly activated by cytosolic lipopolysaccharide (LPS) from Gram-negative bacteria through specific interaction with lipid A moiety in LPS via the CARD domain (Shi et al., 2014). Caspase-4, -5 , and -11 can act on GSDMD to provoke pore opening and drive pyroptosis (Aglietti et al., 2016). Caspase-4, -5, and -11 do not cleave pro-IL-1 $\beta$ and pro-IL-18. However, caspase- $4,-5$, and -11 initiate the formation of the NLRP3 inflammasome possibly by inducing $\mathrm{K}^{+}$efflux caused by GSDMD-NT pores and thus induce pyroptosis (Ruhl and Broz, 2015). IL-1 $\beta$ and IL-18 can be secreted outside of cells through GSDMD-NT pores.

\section{Other Caspases-Induced Pyroptosis}

It has been found that several other caspases operate to induce pyroptosis. For instance, caspase- 3 activated by chemotherapeutic agents could induce pyroptosis in GSDMEexpressing cells (Wang et al., 2017). Active caspase-3 cleaved the central linker of GSDME to release the intramolecular suppression on the N-terminal domain, which subsequently perforated the cell membrane. The formation of GSDME-NT pores triggered cell pyroptosis and resulted in the leakage of cellular components. Moreover, caspase- 8 functions as a modulator of GSDMD-induced pyroptotic cell death. The blockade of transforming growth factor- $\beta$ (TGF- $\beta$ )-activated kinase 1 (TAK1) or the apoptosis inhibitor could foster the receptor-interacting protein kinase 1 (RIPK1)-dependent assembly of the cytosolic caspase-8-activating complex (Orning et al., 2018; Sarhan et al., 2018; Chen K. W. et al., 2019). Active caspase- 8 processed GSDMD to produce the pore-forming fragment (GSDMD-NT). Thus, pyroptotic cell death could proceed under such circumstance. Pyroptosis represents a highly regulated cell death mode that is mediated by GSDMs. A large number of studies have focused on the role of GSDMD in pyroptosis. In recent years, more and more studies have indicated that other GSDM family members also serve as key molecules involved in pyroptosis. The regulatory mechanisms underpinning GSDM-mediated pyroptosis are still not fully clarified. It remains unclear which GSDM family member mainly acts under different conditions. Further work is needed to decipher the upstream signalings that activate GSDMs.

\section{Gasdermin A}

Humans have a single copy of the gene encoding GSDMA, while mice have three copies of the gene (GSDMA1-3) (Tanaka et al., 2013). Human GSDMA is mainly expressed in GI tract, skin, esophagus, stomach and mammary gland (Saeki et al., 2000, 2007; Table 1). GSDMA has been associated with immune-related diseases, such as asthma, inflammatory bowel disease (IBD) and limited cutaneous systemic sclerosis (lcSSc) (Yu et al., 2011; Soderman et al., 2015; Terao et al., 2017). Nevertheless, it remains elusive how GSDMA is involved in the development of immune-related diseases. Additional research is needed to uncover the underlying mechanisms. GSDMA can act as a regulator of programmed cell death. GSDMA was reported to be generally suppressed in gastric cancer cells (Saeki et al., 2007). The expression of GSDMA was coordinated by the transcription factor LIM domain only 
TABLE 1 | The expression and function of gasdermin family members in human.

\begin{tabular}{|c|c|c|c|c|c|}
\hline Human GSDM & Expression pattern & Biological function & Activating enzyme & Disease & References \\
\hline GSDMA & $\begin{array}{l}\text { Gastrointestinal tract, } \\
\text { skin, esophagus, } \\
\text { stomach and mammary } \\
\text { gland }\end{array}$ & Programmed cell death & Not known & $\begin{array}{l}\text { Alopecia and } \\
\text { Infammatory disorders }\end{array}$ & $\begin{array}{l}\text { Saeki et al., 2000, } \\
\text { 2007; Zhou et al., } \\
\text { 2012; Soderman et al., } \\
2015\end{array}$ \\
\hline GSDMB & $\begin{array}{l}\text { Gastrointestinal tract, } \\
\text { lung, lymphocytes, liver, } \\
\text { colon, esophagus and } \\
\text { cancer cells }\end{array}$ & Pyroptosis & $\begin{array}{l}\text { Caspase- } 1,-3,-4,-6, \\
-7 \text { and granzyme A }\end{array}$ & $\begin{array}{l}\text { Asthma, type I } \\
\text { diabetes, ankylosing } \\
\text { spondylitis, } \\
\text { inflammatory disorders } \\
\text { and cancer }\end{array}$ & $\begin{array}{l}\text { Das et al., 2016; Chao } \\
\text { et al., 2017; Chen Q. } \\
\text { et al., 2019; Zhou et al., } \\
2020\end{array}$ \\
\hline GSDMC & $\begin{array}{l}\text { Gastrointestinal tract, } \\
\text { trachea, spleen, } \\
\text { esophagus, stomach } \\
\text { and cancer cells }\end{array}$ & Pyroptosis & Caspase-8 & Cancer & $\begin{array}{l}\text { Kusumaningrum et al., } \\
\text { 2018; Hou et al., } 2020\end{array}$ \\
\hline GSDMD & $\begin{array}{l}\text { Skin, esophagus, } \\
\text { stomach, immune cells } \\
\text { and cancer cells }\end{array}$ & $\begin{array}{l}\text { Pyroptosis, } \\
\text { inflammation, and host } \\
\text { defense }\end{array}$ & $\begin{array}{l}\text { Caspase- } 1,-4,-5,-8, \\
-11 \text {, cathepsin } \mathrm{G} \text { and } \\
\text { neutrophil elastase }\end{array}$ & $\begin{array}{l}\text { Infammatory disorders } \\
\text { and cancer }\end{array}$ & $\begin{array}{l}\text { Katoh and Katoh, } \\
\text { 2004b; Sarhan et al., } \\
\text { 2018; Burgener et al., } \\
\text { 2019; Liu et al., } 2020\end{array}$ \\
\hline GSDME & $\begin{array}{l}\text { Brain, heart, kidney, } \\
\text { placenta and cancer } \\
\text { cells }\end{array}$ & $\begin{array}{l}\text { Pyroptosis and } \\
\text { antitumor immunity }\end{array}$ & $\begin{array}{l}\text { Caspase-3 and } \\
\text { granzyme B }\end{array}$ & Deafness and cancer & $\begin{array}{l}\text { Van Laer et al., 1998; } \\
\text { Rogers et al., 2017; } \\
\text { Zhang et al., } 2020\end{array}$ \\
\hline PJVK (DFNB59) & Auditory system & Not known & Not known & Deafness & $\begin{array}{l}\text { Mujtaba et al., } 2012 ; \\
\text { Liu et al., } 2013\end{array}$ \\
\hline
\end{tabular}

1 (LMO1) and TGF- $\beta$ pathway. GSDMA might mediate TGF$\beta$-induced apoptosis of gastric epithelial cells. GSDMA3 was critical for tumor necrosis factor- $\alpha$ (TNF- $\alpha)$-induced apoptosis pathway in mouse skin keratinocytes by directly upregulating caspase-3 (Lei et al., 2012). Shi P. et al. (2015) revealed that the N-terminal domain of GSDMA3 (GSDMA3-NT) could induce autophagy in human skin kerotinocytes and embryonic kidney cells by reducing mitochondrial activity and promoting the generation of reactive oxygen species (ROS). Consistently, interfering with mitochondrial translocation or ROS generation alleviated GSDMA3-NT-induced cell death (Lin et al., 2015). GSDMA3-NT interacted with membrane lipids (e.g., cardiolipin and phosphoinositide) to form pores, eventually triggering pyroptotic cell death in mammalian cells (Ding et al., 2016).

\section{Gasdermin B}

GSDMB can be detected in GI tract, lung, lymphocytes, liver, colon and the epithelium of the esophagus (Das et al., 2016). Single nucleotide polymorphisms (SNPs) in GSDMB have been shown to be correlated with the increased risk of several diseases, including type I diabetes, ankylosing spondylitis, and inflammatory disorders (Qiu et al., 2013; Ayabe et al., 2016; Hu et al., 2017). Currently, there is a lack of consensus on the cleavage of GSDMB by caspases. The N-terminal domain of GSDMB (GSDMB-NT) was found to induce pyroptosis (Zhou et al., 2020). Moreover, GSDMB failed to trigger pyroptosis in the absence of caspase-1 (Panganiban et al., 2018). It turned out that GSDMB was cleaved by caspase-1 at $\mathrm{Asp}^{236}$. One of the cleaved products was GSDMB-NT that subsequently formed membrane-disrupting pores and executed pyroptosis. Accordingly, the splicing variant (rs11078928) that caused the deletion of 13 amino acids from GSDMB-NT abrogated its pyroptotic activity (Panganiban et al., 2018). Another study revealed that GSDMB combined with the CARD of caspase-4 to induce its oligomerization (Chen Q. et al., 2019). This event triggered conformational alternations to caspase- 4 and increased the enzymatic activity of caspase-4, which fostered GSDMD cleavage to induce cell pyroptosis. Thus, GSDMB indirectly induced non-canonical pyroptosis by elevating caspase- 4 activity. It is possible that the regulatory effects of GSDMB on pyroptotic cell death can be terminated by a negative feedback mechanism, which serves as a critical protective function in the context of the outburst of excessive pyroptosis. Chao et al. (2017) revealed that GSDMB was not a substrate of inflammatory caspases as it lacked the specific cleavage sequence within the interdomain linker region. Intriguingly, apoptotic executioners caspase-3, -6, and -7 were able to cleave GSDMB, separating its $\mathrm{N}$-terminal pore-forming domain from the C-terminal repressor domain. The cleaved N-terminal fragment bound phosphoinositides and cardiolipin, perforated the cell membrane, and eventually evoked cell pyroptosis. This study implied that a cross-talk between the non-canonical pyroptosis pathway and the apoptosis pathway existed. GSDMB might play a role in the transition between different cell death pathways. Further studies should be undertaken to delve into the functional significance of caspase-3, -6, and -7 cleavage within the N-terminal domain of GSDMB. In addition, granzyme A (GZMA) from cytotoxic lymphocytes was found to cleave GSDMB at Lys ${ }^{244}$, which was sufficient to unleash the pore-forming activity of its $\mathrm{N}$-terminal domain (Zhou et al., 2020). GZMA-mediated GSDMB activation subsequently led to pyroptotic cell death in target cells. The GZMA-GSDMB pyroptotic axis may serve a critical role in antimicrobial immunity and cancer pathogenesis. The biological significance of the GZMA-GSDMB pyroptotic pathway awaits further detailed investigation. Collectively, it is important to determine the genuine role of GSDMB in the pyroptosis pathway. 
At present, there is no consensus regarding the role of caspases in the activation of GSDMB. Thus, substantial effort should be placed into revealing the exact mechanisms of GSDMBmediated pyroptosis.

\section{Gasdermin C}

The human genome encodes a single GSDMC, while the mouse genome encodes four homologs known as GSDMC1-4 (Tamura et al., 2007). GSDMC was initially discovered to be highly expressed in metastatic melanoma cells and was referred to as melanoma-derived leucine zipper-containing extranuclear factor (MLZE) (Watabe et al., 2001). Human GSDMC is expressed in GI tract, trachea, spleen, epithelial cells of the esophagus and stomach (Kusumaningrum et al., 2018). Mouse GSDMC is found in the stomach, small intestine, colon and cecum (Tamura et al., 2007). GSDMC acted as a tumor suppressor and exhibited cellgrowth inhibition activity in gastric cancer cells (Saeki et al., 2009). However, GSDMC could have an opposite role in some cancers. For instance, overexpression of GSDMC promoted the proliferation and tumorigenesis of colorectal cancer (CRC) cells (Miguchi et al., 2016). These results bring a contradiction with the involvement of GSDMC in cancer progression. The influence of GSDMC deregulation on carcinogenesis may differ depending on the cancer type. Extensive studies are warranted to completely define the role of GSDMC in cancer. Similar to other GSDMs, the N-terminal domain of GSDMC (GSDMC-NT) was able to induce pyroptosis in human 293T cells (Ding et al., 2016). Recently, Hou et al. (2020) revealed that GSDMC could be specifically cleaved by caspase-8, switching TNF- $\alpha$-induced apoptosis to pyroptosis in breast cancer cells. They found that caspase- 6 could also activate GSDMC. However, only caspase- 8 was activated in the presence of TNF- $\alpha$ plus cycloheximide. This raises an intriguing question of whether GSDMC-NT-induced pyroptosis takes place upon the activation of caspase-6. In addition, the function of GSDMCmediated pyroptosis in cancer is worthy of further investigation.

\section{Gasdermin D}

GSDMD, the best-characterized member of the GSDM family, was identified as a terminal executor of pyroptotic cell death (Wang et al., 2020a). GSDMD is expressed in the skin, esophagus, stomach and immune cells (Katoh and Katoh, 2004b). The C-terminal domain of GSDMD (GSDMDCT) functions to maintain GSDMD molecule in an autoinhibitory conformation. The crystal structure of GSDMD-CT was previously characterized (Kuang et al., 2017). The first loop on GSDMD-CT inserts into the N-terminal domain to stabilize the conformation of full length GSDMD. The autoinhibited conformation can be interrupted once interdomain cleavage by inflammatory caspases. The positive potential surface of GSDMD-NT is exposed and forms high-order oligomers via a charge-charge interaction. The crystal structure of a complex between human caspase-1 and murine GSDMD showed the binding between a loop structure of the GSDMD linker and the active site of caspase-1 (Liu et al., 2020). Of note, GSDMDNT did not bind caspase-1, whereas a hydrophobic pocket within the GSDMD-CT engaged the caspase exosite. This finding suggested that GSDMD-CT acted as a caspase-recruitment module, apart from its auto-inhibitory role. The exact mechanism of GSDMD-pore formation was previously delineated by using atomic force microscopy (Mulvihill et al., 2018). The GSDMDNT initially forms arc-shaped oligomers, which subsequently undergo conversion into slit-shaped oligomers and mature into ring-shaped oligomers. GSDMD-NT generates 16-mer pore complex on the cell membrane, resulting in cell membrane rupture. The lipid-binding feature of GSDMD-NT contributes to its pore-forming ability. It has been reported that GSDMD-NT has high affinity for phosphatidylserine and phosphatidylinositol phosphates, which are restricted to the inner leaflet of the cell membrane, as well as cardiolipin in the inner and outer leaflets of the cell membrane (Liu et al., 2016). GSDMD-NT preferentially binds to phospholipids residing on the inner leaflet of the plasma membrane. Thus, GSDMD-NT kills from within the cell, but does not damage neighboring cells when it is liberated during pyroptotic cell death.

Following the assembly of canonical inflammasome, the activated caspase- 1 cleaves the $53 \mathrm{kDa}$ GSDMD, creating the $31 \mathrm{kDa}$ GSDMD-NT and the $22 \mathrm{kDa}$ GSDMD-CT. GSDMD can also be sheared by murine caspase-11 and human caspase-4/5 at the same site as caspase-1, inducing caspase-1-independent pyroptosis. Moreover, caspase- 8 , cathepsin $G$ and neutrophil elastase (ELANE) are able to proteolytically process GSDMD (Kambara et al., 2018; Orning et al., 2018; Burgener et al., 2019). The liberation of GSDMD-NT induced by inflammatory caspases allows GSDMD-NT to assemble large oligomeric complexes perforating the cell membrane. Following pore formation, cells undergo pyroptotic cell death and their contents are released into the extracellular milieu. It was found that calcium influx through GSDMD pores represented a signal for cells to start membrane repair by recruiting the endosomal sorting complexes required for transport (ESCRT) machinery to damaged membrane regions (Ruhl et al., 2018). Abrogation of the ESCRT-III machinery strongly promoted cell pyroptosis and IL-1 $\beta$ secretion upon canonical or non-canonical inflammasome activation. This study suggested that cells adopted a pro-survival mechanism to limit pyroptosis after inflammasome activation.

GSDMD pores not only induce pyroptotic cell death, but they also play an important role in the inflammasome or caspase-1mediated signaling pathways. GSDMD pores serve as a conduit for extracellular release of mature IL-1 $\beta$ and IL-18. GSDMD depletion significantly inhibited IL-1 $\beta$ secretion upon canonical inflammasome activation, suggesting that GSDMD was necessary for IL-1 $\beta$ release (Karmakar et al., 2015). In certain cell types including human monocytes and mouse bone-marrow-derived macrophages (BMDMs), GSDMD pore-mediated release of IL-1 $\beta$ was even independent of cell lysis (Evavold et al., 2018). Actually, the diameter of the GSDMD pore ranged from approximately 10 to $16 \mathrm{~nm}$, which was wide enough to permit the passage of IL-1 $\beta$ and IL-18 (Sborgi et al., 2016).

The pyroptotic effector GSDMD serves a crucial role in immune response and host defense during pathogen infection. GSDMD-NT can bind to cardiolipin at both the inner and outer membranes of bacteria (Liu et al., 2016). Moreover, ectopic GSDMD-NT was shown to bind and kill extracellular bacteria. By contrast, neither GSDMD-CT nor full length GSDMD 
showed anti-bacterial activity. Activation of inflammasome in bacteria-infected immortalized mouse BMDMs (iBMDMs) induced GSDMD-dependent death of intracellular bacteria. Consequently, the expulsion of viable bacteria from pyroptotic cells was restricted, which could facilitate the control of bacterial infection. Oppositely, GSDMD deficiency attenuated the ability of immune cells to eliminate Neospora caninum (Wang et al., 2020b). In terms of mechanism, GSDMD enhanced IFN- $\gamma$ generation and elicited Thelper type 1 (Th1) immune response against Neospora caninum infection by promoting IL-18 release.

Collectively, GSDMD acts as an essential mediator of pyroptosis and facilitates the release of proinflammatory cytokines. It is still not clear whether cells that actively release cytokines via the GSDMD pore have to proceed to pyroptosis when the quantities of pores achieve a certain threshold. Reportedly, mouse neutrophils did not undergo pyroptosis but possessed the ability to release IL-1 $\beta$ (Chen et al., 2014). The underlying mechanism responsible for the limitation of GSDMDmediated pyroptosis has remained a mystery. Paradoxically, another study indicated that mouse neutrophils underwent GSDMD-mediated pyroptotic cell death (Kambara et al., 2018). Specifically, GSDMD was cleaved by ELANE, resulting in the generation of functionally active GSDMD-NT that caused neutrophil cell death. The factors that control the manner of GSDMD cleavage remain to be identified. The coordination and competition between inflammatory caspases- and ELANEmediated GSDMD activation need further characterization.

Emerging evidence has proven that pyroptosis is capable of eliciting protective immune responses against intracellular pathogens. For instance, GSDMD-mediated pyroptosis causes the release of intracellular pathogens, thereby expelling pathogens from their intracellular replicative niche (Miao et al., 2010a). The exposed pathogens can be killed by innate immune effector cells. Moreover, GSDMD pores mediate the extracellular release of danger signals and proinflammatory cytokines that recruit immune cells to the site of infection, leading to the elimination of pathogens. Pyroptosis could facilitate the generation of pore-induced intracellular traps (PITs) (Jorgensen et al., 2016). PITs helped to capture intracellular bacteria and contributed to their clearance by efferocytosis. In parallel, pyroptosis removed bacteria-infected cells from murine intestinal epithelium in a caspase-dependent manner (Knodler et al., 2014). Pathogen exclusion, immune cell recruitment, PIT formation and the clearance of infected cells via pyroptosis constitute multifaceted immune defenses that function to provide host protection. Unsurprisingly, the pyroptotic executioner GSDMD takes part in host immune defense. Activated GSDMD was reported to attack bacteria and attenuate their viability (Liu et al., 2016). Considering that other molecules involved in the pyroptosis pathway (e.g., cytokines and caspases) can induce anti-microbial responses, the genuine contribution of GSDMD to pyroptosis-mediated host defense against pathogen invasion merits further study.

\section{Gasdermin E}

GSDME, initially identified as DFNA5, can be detected in the brain, heart, kidney and placenta (Webb et al., 2007). In general, GSDME is highly expressed in normal cells, whereas its expression is varied in different types of cancer (Croes et al., 2017). Since its discovery in 1998, a large number of studies have revealed the potential implication of GSDME in cancer (Akino et al., 2007; Kim et al., 2008; Ibrahim et al., 2019a). It was reported that GSDME could significantly inhibit cell growth and colony-forming ability in CRC cells (Kim et al., 2008). GSDME suppressed the proliferation of hepatocellular carcinoma (HCC) cells by inducing cell cycle arrest (Wang et al., 2013). GSDME-deficient melanoma cells formed and grew larger tumors in vivo compared to control melanoma cells (Rogers et al., 2019). Another study showed that forced expression of GSDME enhanced the susceptibility of drug-resistant melanoma cells to therapeutic drugs by inducing caspase-3-mediated apoptosis (Lage et al., 2001). Based on the given evidence, GSDME function as a tumor suppressor.

GSDME has been identified to be involved in programmed cell death, including pyroptosis and apoptosis. GSDME-expressing cells were prone to suffer pyroptosis, when stimulated with apoptotic stimuli (Rogers et al., 2017). GSDME served as a physiological target for caspase-3 after its activation by the apoptotic protease-activating factor 1 (Apaf-1) apoptosome. Caspase-3 cleaved GSDME after Asp ${ }^{270}$ to generate the necrotic N-terminal fragment of GSDME (GSDME-NT). GSDME-NT targeted the cellular membrane to form large pores, eventually inducing cell pyroptosis. Meanwhile, GSDME-NT permeabilized the mitochondrial membrane, resulting in the release of cytochrome c (cyto c) and activation of apoptosome (Rogers et al., 2019). Thus, GSDME was capable of augmenting the mitochondrial apoptotic pathway.

The characterization of GSDM expression and function is an intriguing area for future research. It is yet to be defined if proteolytic cleavage is a common requirement to liberate the $\mathrm{N}$-terminal functional domain of GSDMs. Continued studies are required to identify the premier signals that govern GSDM activation. The profound impacts of GSDM-mediated pyroptosis on immunity, disease and beyond should be adequately explored. A better comprehension of the cellular function of GSDMs could provide potential therapeutic avenues for the treatment of human diseases.

\section{THE ROLE OF GASDERMINS IN CANCER PATHOGENESIS}

Based on previous literatures on human cancers, researchers have revealed that GSDMs are deregulated in cancer tissues, including CRC, gastric cancer (GC), non-small cell lung cancer (NSCLC), breast cancer, colon adenocarcinoma, adenoid cystic carcinoma (ACC) and cervical squamous cell carcinoma (CSCC). GSDMs have been shown to regulate cancer cell proliferation, apoptosis, invasion, metastasis and chemoresistance (Figure 2). Activated GSDMs act as downstream effectors in the pyroptosis pathway. The induction of pyroptosis could promote cancer cell death. Moreover, pore-forming GSDMs modulate antitumor immunity. GSDMs may act as oncogenes or tumor suppressors that participate in the initiation and development of cancer, 
which put forward a research direction of therapeutic targets for cancer intervention. In addition, GSDMs may hold great promise as prospective biomarkers for early screening, evaluation of therapeutic efficacy and prediction of clinical outcomes in cancer patients.

\section{Gasdermins Regulate Cancer Cell Proliferation}

GSDMC was shown to be upregulated in CRC tissues relative to that in corresponding adjacent normal tissues (Miguchi et al., 2016). GSDMC promoted the carcinogenesis and proliferation of CRC cells. It also facilitated xenograft tumor growth in vivo. GSDMC might represent a viable therapeutic target for CRC treatment. GSDMD was downregulated in GC cells and tissues compared with the controls (Wang et al., 2018a). The reduced expression of GSDMD significantly facilitated the proliferation of GC cells partially by activating extracellular signal-regulated kinase (ERK), signal transducer and activator of transcription 3 (STAT3), and phosphatidylinositol 3-kinase (PI3K)/protein kinase B (Akt) signaling pathways and modulating cell cyclerelated proteins. These findings shed new light on the relationship between GSDMD and GC development.

\section{Gasdermins Contribute to Cancer Cell Death}

STAT3 could be activated by phosphorylation at $\mathrm{Tyr}^{705}$ (STAT3Y705) in response to hypoxia (Pawlus et al., 2014). Under hypoxia, STAT3-Y705 interacted with the important immunecheckpoint programmed death-ligand 1 (PD-L1) and favored its nuclear translocation, hence enhancing GSDMC transcription (Hou et al., 2020). GSDMC was then cleaved by caspase- 8 in breast cancer cells treated with TNF- $\alpha$. Caspase- 8 induced the opening of the GSDMC-NT pore on the cell membrane, leading to pyroptosis in breast cancer cells. Therefore, caspase-8/GSDMC initiated a non-canonical pyroptosis pathway in breast cancer cells. GSDMD was markedly upregulated in NSCLC compared with paired neighboring normal tissues (Gao et al., 2018). High expression of GSDMD was linked with malignant characteristics and poor prognosis in NSCLC patients. Depletion of GSDMD suppressed the proliferation of NSCLC cells by favoring apoptosis and blocking the epidermal growth factor receptor (EGFR)/Akt signaling pathway. In GSDMD-silenced NSCLC cells, activation of the pyroptotic signaling (NLRP3/caspase-1) induced apoptotic cell death, instead of pyroptosis. In term of mechanisms, GSDMD knockdown activated the cleavage of caspase- 3 and poly(ADP-ribose) polymerase (PARP), and facilitated the death of NSCLC cells via intrinsic mitochondrial apoptotic pathways. Likewise, overexpression of GSDMDNT caused death of human schwannoma cells in vitro (Ahmed et al., 2019). Intratumoral injection of adeno-associated serotype 1 virus vectors containing GSDMD-NT transgene (AAV1-rP0-GSDMDNterm) promoted pyroptosis and repressed tumor growth in both mouse schwannoma allograft tumors and human schwannoma xenografts. This treatment did not cause any neuronal toxicity. The AAV1-rP0-GSDMDNterm vector might provide an effective therapeutic approach for schwannoma treatment.

The expression of GSDME was observed in some cancers, while it was epigenetically silenced in the majority of cancer cell lines owing to the hypermethylation of its promoter (Croes et al., 2019). GSDME could switch caspase-3-dependent chemotherapy drug-induced apoptosis into pyroptosis (Yu et al., 2019; Zhang et al., 2019). The mode of cancer cell death (apoptosis or pyroptosis) depended on the expression level of GSDME. GSDME-silenced Jurkat cells underwent apoptotic cell death following treatment with diverse chemotherapeutic agents (Wang et al., 2017). By contrast, GSDME-expressing SHSY5Y neuroblastoma, MeWo melanoma and NCI-H522 lung cancer cells developed pyroptotic responses to chemotherapeutic drugs. Moreover, the caspase inhibitor or depletion of GSDME blocked chemotherapeutic drug-induced pyroptosis in cancer cells. Overexpression of GSDME in GSDME-deficient HeLa cells switched anticancer drug-induced apoptosis to pyroptosis in a caspase-3-dependent manner. As GSDME was expressed in many normal tissues, chemotherapeutic agents caused the death of normal human cells through GSDME-mediated pyroptosis (Wang et al., 2017). The expression of GSDME was detected in human lung cancer cells (Zhang et al., 2019). The chemotherapeutic agent cisplatin triggered pyroptotic cell death in lung cancer cells by activating the caspase-3/GSDME pathway. Accordingly, knockdown of GSDME remarkably suppressed cisplatin-induced pyroptosis in lung cancer cells. GSDME expression was markedly decreased in human breast cancer and colon adenocarcinoma tissues compared with matched tumor-adjacent tissues mainly attributing to its hypermethylation (Fan et al., 2019). The DNA methyltransferase inhibitor decitabine (DAC) could increase the expression of GSDME by demethylation in mouse breast cancer and colon carcinoma cells. The reversal of GSDME silencing by DAC sensitized cancer cells with chemotherapeutic drugs. Chemotherapy activated caspase-3 for processing GSDME into GSDME-NT, eventually inducing pyroptosis in breast cancer and colon carcinoma cells. Collectively, the combination of GSDME activators and chemotherapeutics might be effective approaches for controlling human cancers. However, GSDME-mediated pyroptosis has been proposed as an underlying mechanism of chemotherapy-induced side effects. The effectiveness and safety of GSDME-based anticancer therapeutics must be validated before its translation into clinical practice.

\section{Gasdermins Affect Cancer Cell Invasion and Metastasis}

A previous report showed that the expression level of GSDMB was higher in breast cancer tissues than normal breast tissues (Hergueta-Redondo et al., 2014). Upregulation of GSDMB was related to poor outcome and increased metastasis in breast cancer patients. Overexpression of GSDMB was capable of enhancing the motility and invasion of breast cancer cells. Oppositely, depletion of GSDMB inhibited the migration and invasion of breast cancer cells. It was likely that the activation of Ras-related C3 botulinum toxin substrate $1(\mathrm{Rac}-1)$ and cell division cycle 


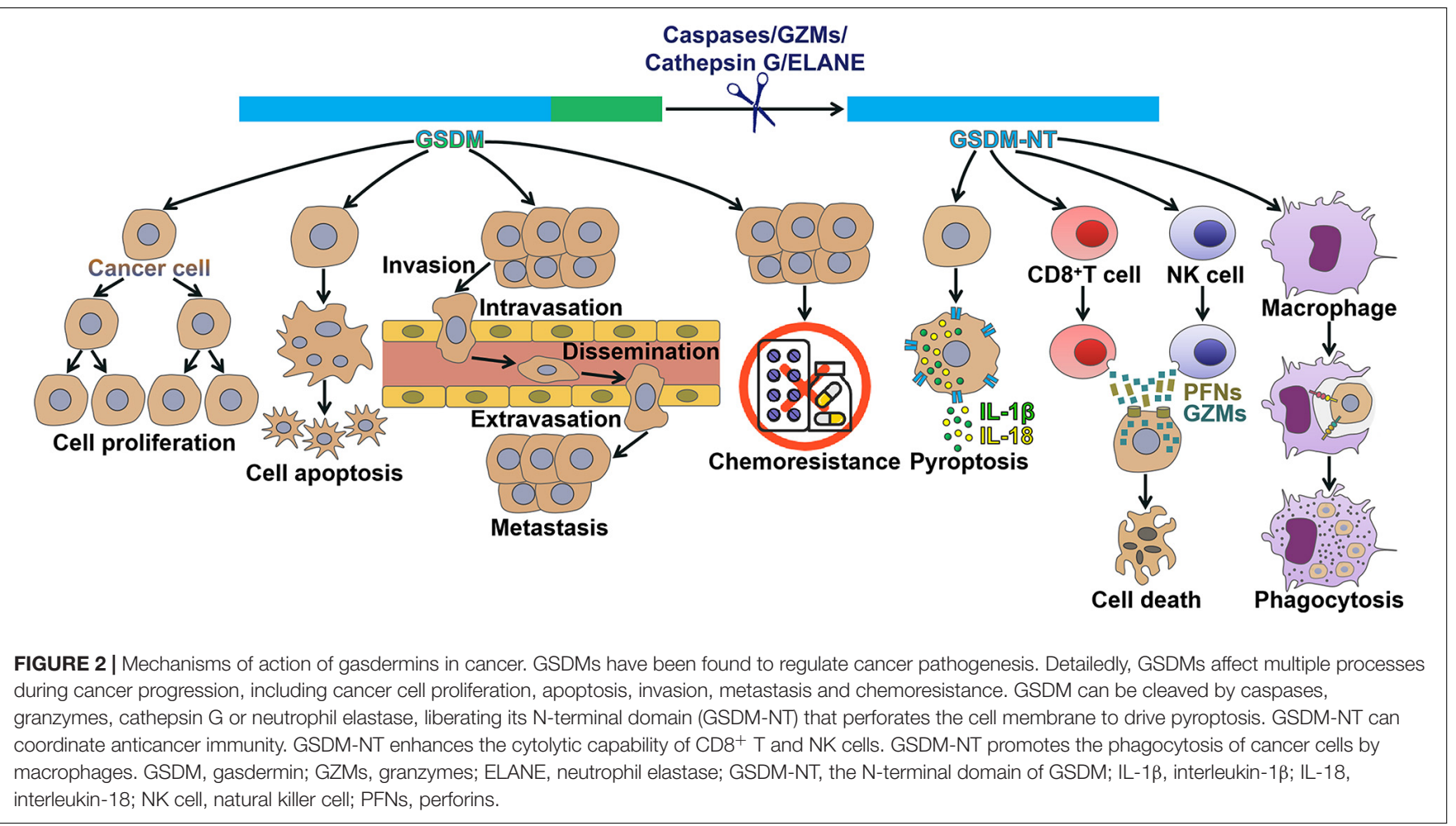

42 (Cdc-42) GTPases partially mediated the pro-migratory and pro-invasive function of GSDMB. Furthermore, GSDMB could promote tumor progression and metastasis in mouse xenograft models. Therefore, GSDMB might serve as a potential therapeutic target for the treatment of breast cancer. The expression level of GSDMD was markedly higher in ACC tissues than that of corresponding adjacent non-cancerous tissues (Shen et al., 2020). Moreover, high expression of GSDMD enhanced the invasive capacity of ACC cells. It was likely that GSDMD could be an indicator of ACC invasiveness and aggressiveness.

\section{Gasdermins Orchestrate Chemoresistance in Cancer Cells}

GSDMB overexpression showed the potential to predict poor clinical outcome in patients with human epidermal growth factor receptor 2 (HER2)-positive breast cancer (Hergueta-Redondo et al., 2016). Moreover, GSDMB upregulation was associated with reduced therapeutic responses and tumor metastasis in patients with HER2-positive breast cancer. GSDMB markedly increased cell survival to trastuzumab treatment in HER2-positive breast cancer cells. Likewise, GSDMB was correlated with trastuzumab resistance phenotype in breast cancer patients derived xenografts. To summarize, GSDMB might act as a new biomarker in HER2positive breast cancer, opening up new opportunities for efficient anticancer therapies. The efficacy of GSDMB-targeting therapies was studied in mice bearing HER2 breast cancer xenografts (Molina-Crespo et al., 2019). Specifically, intracellular delivery of the anti-GSDMB antibody by nanocapsules could inhibit tumor growth and metastasis in vivo. Additionally, in vitro evidence also showed that the anti-GSDMB nanotherapy was able to sensitize
GSDMB-expressing breast cancer cells to trastuzumab treatment. Therefore, reducing the expression of GSDMB may be a potential therapeutic option for HER2-positive breast cancer.

\section{Gasdermins Modulate Antitumor Immunity}

The cleaved GSDMB show an anti-carcinogenic capability. GZMmediated cell death constitutes a pivotal mechanism for cytotoxic T lymphocytes (CTLs) to remove tumor cells (Pardo et al., 2008). Natural killer (NK) cells and CTLs triggered pyroptotic cell death in GSDMB-positive cells (Zhou et al., 2020). Mechanistically, GSDMB was proteolytically processed by lymphocyte-derived GZMA, thus causing pyroptosis in target cells. Enhancing GZMA expression induced pyroptosis in GSDMB-expressing esophageal carcinoma, rectum adenocarcinoma and colorectal adenocarcinoma cells. Introducing GZMA-cleavable GSDMB into colon carcinoma and melanoma cells almost completely restrained tumor growth in mice. These findings demonstrated that GSDMB-dependent pyroptosis formed a CTL-mediated killing mechanism, which might contribute to enhancing antitumor immunity. GSDMD was positively associated with $\mathrm{CD}^{+} \mathrm{T}$ cell markers in NSCLC samples (Xi et al., 2019). In activated human $\mathrm{CD}^{+} \mathrm{T}$ cells, GSDMD was cleaved by caspase-4 and -11. GSDMD, mainly in cleaved form, was highly expressed in activated $\mathrm{CD}^{+} \mathrm{T}$ lymphocytes. Remarkably, GSDMD silencing caused decreased cytolytic capability of CD8 ${ }^{+}$ $\mathrm{T}$ cells on NSCLC cells. These observations suggested that activated GSDMD might play a role in inducing CTL responses to NSCLC cells. GSDMD may be crucial for establishing an inflammatory microenvironment around cancer cells. The 
involvement of GSDMD in anticancer immunity is another topic that needs further investigation. Considerable efforts should be dedicated to uncovering the detail function of GSDMD in cancer.

GSDME contributes to tumor suppression by activating antitumor immunity. Ectopic expression of GSDME in breast cancer and melanoma cells markedly repressed tumor growth in mice (Zhang et al., 2020). Specifically, overexpression of GSDME enhanced immune responses within tumors. GSDMEexpressing tumors had more tumor-infiltrating immune cells, including NK cells, tumor-associated macrophages (TAMs) and $\mathrm{CD}^{+} \mathrm{T}$ lymphocytes, in comparison with control tumors. The immune cells in GSDME-expressing tumors produced more perforins (PFNs), GZMB and cytokines upon activation. Moreover, both $\mathrm{NK}$ and $\mathrm{CD}^{+}{ }^{+} \mathrm{T}$ cells were required for the suppressive effect of GSDME on breast cancer and melanoma cells. GSDME boosted TAM phagocytosis and the functions of tumor-infiltrating immune cells. Upregulation of GSDME had no remarkable impact on tumor growth in killer lymphocytedepleted or PFN-deficient mice. Thus, GSDME-mediated tumor suppression was attributed to cytotoxic lymphocyte killing. Of note, cleaved GSDME played a key role in strengthening tumor immunosuppression (Zhang et al., 2020). Specifically, killer cell GZMB directly cleaved GSDME at the same site as caspase-3 $\left(\mathrm{Asp}^{270}\right)$ and induced caspase-independent pyroptosis in breast cancer and melanoma cells. By contrast, non-cleavable or poredefective GSDME did not exhibit tumor suppressive ability. A recent study suggested that blockade of the ERK1/2 pathway by targeted inhibitors could induce GSDME-mediated pyroptosis in melanoma cells (Erkes et al., 2020). Markedly, GSDME pores mediated the leakage of DAMPs including high mobility group protein B1 (HMGB1) from the melanoma cells. The released DAMPs then caused the activation of dendritic cells (DCs) and, in turn, enhanced antitumor T cell activity. Therefore, GSDMEdependent pyroptosis form an important mechanism involved in the antitumor activity of ERK1/2 pathway inhibitors.

\section{Gadermins Show the Potential as Cancer Biomarkers}

Lutkowska et al. (2017) discovered that a SNP located $9.5 \mathrm{~kb}$ downstream of the GSDMB gene was linked with invasive cervical cancer. They further examined the impact of rs8067378 genotype on GSDMB expression level in non-cancerous and CSCC tissues. As a result, the rs 8067378 polymorphism increased GSDMB expression, which was significantly associated with tumor development and dissemination in CSCC patients. GSDMB polymorphism might act as an indicative biomarker for CSCC progression. The expression of GSDMD was evidently different between 108 cases of breast cancer tissues and 23 cases of para-cancerous benign tissues (Wu et al., 2020). The GSDMD expression level was inversely correlated with the clinical stage, the pathologic grade of tumor tissues, tumor size and metastasis in patients with breast cancer. The high expression of GSDMD was linked to longer overall survival in patients. These finding implied that GSDMD might be involved in the invasion, metastasis and prognosis of breast cancer.
The clinical utility of GSDMD as a prognostic factor and a genuine therapeutic target for breast cancer should be adequately investigated in future studies.

Previously, the methylation patterns of the GSDME gene across fourteen distinct tumor types were analyzed through The Cancer Genome Atlas (TCGA) methylation data (Ibrahim et al., 2019b). The methylation patterns of GSDME displayed significant variation between cancerous and normal tissues. GSDME possessed unique methylation patterns across different tumors. For instance, uterine carcinomas had the highest count of hypomethylated GSDME GpGs, followed by breast, colorectal and renal clear cell cancers. Colorectal and breast cancers, followed by lung and prostate cancers, had the greatest count of hypermethylated CpGs. GSDME methylation had the capacity to discriminate between different tumor types. GSDME methylation patterns might represent useful detection biomarkers in both a pan-cancer and tumor-specific context. Similarly, differential methylations in GSDME CpGs were found between CRC and normal tissues (Ibrahim et al., 2019a). The combination of two CpGs (CpG4 residing within the gene body and CpG12 residing in the putative promoter region) could differentiate CRC tissues from normal tissues with high accuracy. These findings suggested that GSDME could be exploited as a prospective biomarker for CRC screening.

\section{CONCLUSION AND FUTURE PERSPECTIVES}

GSDMs are involved in the regulation of host immune responses and cell growth. They exert different effects on cell growth. For instance, GSDMA, GSDMC, GSDMD, and GSDME exert inhibitory effects on cell growth, while GSDMB act as an oncoprotein in cancer. Moreover, the N-terminal fragments of GSDMA-E can oligomerize to form pores in the plasma membrane and thus serve as executors for pyroptotic cell death. GSDM-mediated pyroptosis may foster cancer cell death and have anticancer effects. Based on the given evidence, GSDMs could be used as prospective molecular targets for developing effective treatments of human cancers. However, the studies on GSDM biology only touch the tip of the iceberg. Many questions have yet to be answered. First, it is important to unravel the exact mechanisms responsible for the activation of different GSDMs. The molecules involving in GSDM regulation may be potential pharmaceutical targets for the treatment of cancer. Secondly, the derivation of active GSDMs and their biological significance warrant further study. Caspases, GZMs, cathepsin G and ELANE have been found to cleave GSDMs. It is important to investigate whether GSDMs can be activated by other molecules. Active GSDMs play a role in inflammation and cell death. They are correlated with inflammatory disorders and cancers. The relationship between GSDMs and diseases could be a future research direction. Thirdly, it is intriguing how GSDMs take part in pyroptosis mechanistically. The initiation mechanism is the key to GSDMs-mediated pyroptosis. The stimulating factors that activate caspases or beyond during the original 
stage of the pyroptosis program are required to be determined. Fourthly, GSDM pores function as a conduit for the release of intracellular contents, such as proinflammatory cytokines, alarmins, endogenous ligands and DAMPs. These findings raise an intriguing question concerning the functional significance of GSDM pores in the communication between cancer cells and their microenvironment. Further study is warranted to verify this hypothesis. Fifthly, it is crucial to illuminate the abundance and activation of GSDMs in different types of cancer cells. GSDMs have been shown to be aberrantly expressed in cancer, suggesting that they may be utilized as novel biomarkers for cancer detection. However, the expression profile of GSDMs in cancer cells/tissues is still poorly delineated. As the activation of GSDMs is highly regulated, significant work is justified to understand which GSDM family members can be activated in certain forms of cancer. Moreover, it is still not clear whether the activation of GSDMs is cell-type specific. Comprehensive investigation of GSDM expression and methylation in cancer patients will open up new opportunities for cancer diagnosis and prevention. Finally, GSDMs may show synergistic or antagonistic effects on cancer cells. Therefore, the detailed functions of different GSDMs in cancer are still not well understood and remain to be extensively studied.

Owing to the important function of GSDMs in pyroptosis and cancer progression, pharmacological coordination of GSDM activity may have the potential to become an effective anticancer therapeutic approach. At present, the characterization of specific GSDM regulators has just started. A previous report indicated that nigericin could activate NLRP3 inflammasome in LPSprimed macrophages and facilitated the recruitment of GSDMD to NLRP3, thereby inducing pyroptosis (He et al., 2015). Glutathione peroxidase 4 (GPX4) was able to modulate GSDMD activity (Kang et al., 2018). Mechanistically, GPX4 inhibited cellular lipid peroxidation in murine myeloid cells that prevented GSDMD activation and GSDMD-NT pore formation. Recently, Humphries et al. (2020) revealed that fumarate acted as a pyroptosis blocker. Fumarate-induced succination of human

\section{REFERENCES}

Aglietti, R. A., Estevez, A., Gupta, A., Ramirez, M. G., Liu, P. S., Kayagaki, N., et al. (2016). GsdmD p30 elicited by caspase-11 during pyroptosis forms pores in membranes. Proc. Natl. Acad. Sci. U.S.A. 113, 7858-7863. doi: 10.1073/pnas. 1607769113

Ahmed, S. G., Abdelanabi, A., Doha, M., and Brenner, G. J. (2019). Schwannoma gene therapy by adeno-associated virus delivery of the pore-forming protein Gasdermin-D. Cancer Gene Ther. 26, 259-267. doi: 10.1038/s41417-0180077-3

Akino, K., Toyota, M., Suzuki, H., Imai, T., Maruyama, R., Kusano, M., et al. (2007). Identification of DFNA5 as a target of epigenetic inactivation in gastric cancer. Cancer Sci. 98, 88-95. doi: 10.1111/j.1349-7006.2006.00351.x

Ayabe, T., Fukami, M., Ogata, T., Kawamura, T., Urakami, T., Kikuchi, N., et al. (2016). Variants associated with autoimmune type 1 diabetes in Japanese children: implications for age-specific effects of cis-regulatory haplotypes at 17q12-q21. Diabet. Med. 33, 1717-1722. doi: 10.1111/dme.13175

Ball, D. P., Taabazuing, C. Y., Griswold, A. R., Orth, E. L., Rao, S. D., Kotliar, I. B., et al. (2020). Caspase-1 interdomain linker cleavage is required for pyroptosis. Life Sci. Alliance 3:e202000664. doi: 10.26508/lsa.202000664
GSDMD at Cys $^{191}$ prevented its activation by caspase-1, oligomerization and ability to trigger cell death. These studies highlight the potential for treatment of human malignancies with GSDMD regulators. Nevertheless, promising therapies aiming to target GSDM activity are still underdeveloped. Considerable efforts must be implemented to identify GSDM-targeted molecules in pursuit of new drug development. Nevertheless, the GSDM pores facilitate the release of proinflammatory contents from the cell, which signifies the inflammatory potential of pyroptosis. The use of GSDM inducer may causes unfavorable outcomes in cancer patients. It is likely that excessive induction of GSDM-mediated pyroptosis exacerbates the inflammatory responses, and thus causes inflammationdriven disorders. Accordingly, the effectiveness and side effect of GSDM inducers must be thoroughly examined. It should be noted that GSDMB can promote cancer growth. Elevation of GSDMB expression/activity may offer a favorable niche for carcinogenesis and cancer progression. Therefore, the authentic effect of GSDM activation on cancer pathogenesis requires to be ascertained. Further insights into the roles of GSDMs in cancer will not only improve our understanding of the intricate mechanisms underlying cancer pathogenesis but will also help to exploit new therapeutic approaches for cancer treatment.

\section{AUTHOR CONTRIBUTIONS}

MW conceived this study, prepared the figures, and drafted the manuscript. XC collected the related manuscripts. YZ revised the manuscript. All authors reviewed and approved the final manuscript.

\section{FUNDING}

This work was supported by the National Natural Science Foundation of China (No. 81701991) and the Applied Basic Research Program of Qingdao, China (No. 17-1-1-59-jch).

Bergsbaken, T., and Cookson, B. T. (2007). Macrophage activation redirects yersinia-infected host cell death from apoptosis to caspase-1-dependent pyroptosis. PLoS Pathog. 3:e161. doi: 10.1371/journal.ppat.0030161

Broz, P., von Moltke, J., Jones, J. W., Vance, R. E., and Monack, D. M. (2010). Differential requirement for Caspase-1 autoproteolysis in pathogen-induced cell death and cytokine processing. Cell Host Microbe 8, 471-483. doi: 10.1016/ j.chom.2010.11.007

Burgener, S. S., Leborgne, N. G. F., Snipas, S. J., Salvesen, G. S., Bird, P. I., and Benarafa, C. (2019). Cathepsin G inhibition by Serpinb1 and Serpinb6 prevents programmed necrosis in neutrophils and monocytes and reduces GSDMD-driven inflammation. Cell Rep. 27, 3646-3656.e3645.

Case, C. L., Kohler, L. J., Lima, J. B., Strowig, T., de Zoete, M. R., Flavell, R. A., et al. (2013). Caspase-11 stimulates rapid flagellin-independent pyroptosis in response to Legionella pneumophila. Proc. Natl. Acad. Sci. U.S.A. 110, 18511856. doi: 10.1073/pnas.1211521110

Chang, Y., Zhu, J., Wang, D., Li, H., He, Y., Liu, K., et al. (2020). NLRP3 inflammasome-mediated microglial pyroptosis is critically involved in the development of post-cardiac arrest brain injury. J. Neuroinflammation 17:219.

Chao, K. L., Kulakova, L., and Herzberg, O. (2017). Gene polymorphism linked to increased asthma and IBD risk alters gasdermin-B structure, a sulfatide and 
phosphoinositide binding protein. Proc. Natl. Acad. Sci. U.S.A. 114, E1128E1137.

Chen, K. W., Demarco, B., Heilig, R., Shkarina, K., Boettcher, A., Farady, C. J., et al. (2019). Extrinsic and intrinsic apoptosis activate pannexin-1 to drive NLRP3 inflammasome assembly. EMBO J. 38:e101638.

Chen, K. W., Gross, C. J., Sotomayor, F. V., Stacey, K. J., Tschopp, J., Sweet, M. J., et al. (2014). The neutrophil NLRC4 inflammasome selectively promotes IL1beta maturation without pyroptosis during acute Salmonella challenge. Cell Rep. 8, 570-582. doi: 10.1016/j.celrep.2014.06.028

Chen, K. W., Monteleone, M., Boucher, D., Sollberger, G., Ramnath, D., Condon, N. D., et al. (2018). Noncanonical inflammasome signaling elicits gasdermin D-dependent neutrophil extracellular traps. Sci. Immunol. 3:eaar6676. doi: 10.1126/sciimmunol.aar6676

Chen, Q., Shi, P., Wang, Y., Zou, D., Wu, X., Wang, D., et al. (2019). GSDMB promotes non-canonical pyroptosis by enhancing caspase- 4 activity. J. Mol. Cell Biol. 11, 496-508. doi: 10.1093/jmcb/mjy056

Chen, X., He, W. T., Hu, L., Li, J., Fang, Y., Wang, X., et al. (2016). Pyroptosis is driven by non-selective gasdermin-D pore and its morphology is different from MLKL channel-mediated necroptosis. Cell Res. 26, 1007-1020. doi: 10.1038/cr. 2016.100

Cookson, B. T., and Brennan, M. A. (2001). Pro-inflammatory programmed cell death. Trends Microbiol. 9, 113-114. doi: 10.1016/s0966-842x(00)01936-3

Croes, L., de Beeck, K. O., Pauwels, P., Vanden Berghe, W., Peeters, M., Fransen, E., et al. (2017). DFNA5 promoter methylation a marker for breast tumorigenesis. Oncotarget 8, 31948-31958. doi: 10.18632/oncotarget.16654

Croes, L., Fransen, E., Hylebos, M., Buys, K., Hermans, C., Broeckx, G., et al. (2019). Determination of the potential tumor-suppressive effects of gsdme in a chemically induced and in a genetically modified intestinal cancer mouse model. Cancers 11:1214. doi: 10.3390/cancers11081214

Das, S., Miller, M., and Broide, D. H. (2017). Chromosome 17q21 Genes ORMDL3 and GSDMB in asthma and immune diseases. Adv. Immunol. 135, 1-52. doi: 10.1016/bs.ai.2017.06.001

Das, S., Miller, M., Beppu, A. K., Mueller, J., McGeough, M. D., Vuong, C., et al. (2016). GSDMB induces an asthma phenotype characterized by increased airway responsiveness and remodeling without lung inflammation. Proc. Natl. Acad. Sci. U.S.A. 113, 13132-13137. doi: 10.1073/pnas.1610433113

Delmaghani, S., del Castillo, F. J., Michel, V., Leibovici, M., Aghaie, A., Ron, U., et al. (2006). Mutations in the gene encoding pejvakin, a newly identified protein of the afferent auditory pathway, cause DFNB59 auditory neuropathy. Nat. Genet. 38, 770-778. doi: 10.1038/ng1829

Demarco, B., Grayczyk, J. P., Bjanes, E., Le Roy, D., Tonnus, W., Assenmacher, C. A., et al. (2020). Caspase-8-dependent gasdermin D cleavage promotes antimicrobial defense but confers susceptibility to TNF-induced lethality. Sci. Adv. 6:eabc3465. doi: 10.1126/sciadv.abc3465

Ding, J., Wang, K., Liu, W., She, Y., Sun, Q., Shi, J., et al. (2016). Pore-forming activity and structural autoinhibition of the gasdermin family. Nature 535, 111-116. doi: 10.1038/nature18590

Dubois, H., Sorgeloos, F., Sarvestani, S. T., Martens, L., Saeys, Y., Mackenzie, J. M., et al. (2019). Nlrp3 inflammasome activation and Gasdermin D-driven pyroptosis are immunopathogenic upon gastrointestinal norovirus infection. PLoS Pathog. 15:e1007709. doi: 10.1371/journal.ppat.1007709

Ebermann, I., Walger, M., Scholl, H. P., Charbel Issa, P., Luke, C., Nurnberg, G., et al. (2007). Truncating mutation of the DFNB59 gene causes cochlear hearing impairment and central vestibular dysfunction. Hum. Mutat. 28, 571-577. doi: 10.1002/humu.20478

Erkes, D. A., Cai, W., Sanchez, I. M., Purwin, T. J., Rogers, C., Field, C. O., et al. (2020). Mutant BRAF and MEK inhibitors regulate the tumor immune microenvironment via pyroptosis. Cancer Discov. 10, 254-269. doi: 10.1158/ 2159-8290.cd-19-0672

Evavold, C. L., Ruan, J., Tan, Y., Xia, S., Wu, H., and Kagan, J. C. (2018). The pore-forming protein gasdermin $\mathrm{D}$ regulates interleukin-1 secretion from living macrophages. Immunity 48, 35-44.e36.

Fan, J. X., Deng, R. H., Wang, H., Liu, X. H., Wang, X. N., Qin, R., et al. (2019). Epigenetics-based tumor cells pyroptosis for enhancing the immunological effect of chemotherapeutic nanocarriers. Nano Lett. 19, 8049-8058. doi: 10. 1021/acs.nanolett.9b03245

Fernandes-Alnemri, T., Wu, J., Yu, J. W., Datta, P., Miller, B., Jankowski, W., et al. (2007). The pyroptosome: a supramolecular assembly of ASC dimers mediating inflammatory cell death via caspase-1 activation. Cell Death. Differ. 14, 1590-1604. doi: 10.1038/sj.cdd.4402194

Fernandes-Alnemri, T., Yu, J. W., Datta, P., Wu, J., and Alnemri, E. S. (2009). AIM2 activates the inflammasome and cell death in response to cytoplasmic DNA. Nature 458, 509-513. doi: 10.1038/nature07710

Fink, S. L., and Cookson, B. T. (2006). Caspase-1-dependent pore formation during pyroptosis leads to osmotic lysis of infected host macrophages. Cell Microbiol. 8, 1812-1825. doi: 10.1111/j.1462-5822.2006.00751.x

Gao, J., Qiu, X., Xi, G., Liu, H., Zhang, F., Lv, T., et al. (2018). Downregulation of GSDMD attenuates tumor proliferation via the intrinsic mitochondrial apoptotic pathway and inhibition of EGFR/Akt signaling and predicts a good prognosis in nonsmall cell lung cancer. Oncol. Rep. 40, 1971-1984.

Guey, B., Bodnar, M., Manie, S. N., Tardivel, A., and Petrilli, V. (2014). Caspase-1 autoproteolysis is differentially required for NLRP1b and NLRP3 inflammasome function. Proc. Natl. Acad. Sci. U.S.A. 111, 17254-17259. doi: 10.1073/pnas.1415756111

Hari, A., Zhang, Y., Tu, Z., Detampel, P., Stenner, M., Ganguly, A., et al. (2014). Activation of NLRP3 inflammasome by crystalline structures via cell surface contact. Sci. Rep. 4:7281.

Harton, J. A., Linhoff, M. W., Zhang, J., and Ting, J. P. (2002). Cutting edge: CATERPILLER: a large family of mammalian genes containing CARD, pyrin, nucleotide-binding, and leucine-rich repeat domains. J. Immunol. 169, 40884093. doi: 10.4049/jimmunol.169.8.4088

He, W. T., Wan, H., Hu, L., Chen, P., Wang, X., Huang, Z., et al. (2015). Gasdermin $\mathrm{D}$ is an executor of pyroptosis and required for interleukin-1beta secretion. Cell Res. 25, 1285-1298. doi: 10.1038/cr.2015.139

Heil, F., Hemmi, H., Hochrein, H., Ampenberger, F., Kirschning, C., Akira, S., et al. (2004). Species-specific recognition of single-stranded RNA via toll-like receptor 7 and 8. Science 303, 1526-1529. doi: 10.1126/science.1093620

Hergueta-Redondo, M., Sarrio, D., Molina-Crespo, A., Megias, D., Mota, A., RojoSebastian, A., et al. (2014). Gasdermin-B promotes invasion and metastasis in breast cancer cells. PLoS One 9:e90099. doi: 10.1371/journal.pone.0090099

Hergueta-Redondo, M., Sarrio, D., Molina-Crespo, A., Vicario, R., BernadoMorales, C., Martinez, L., et al. (2016). Gasdermin B expression predicts poor clinical outcome in HER2-positive breast cancer. Oncotarget 7, 56295-56308. doi: 10.18632/oncotarget.10787

Hornung, V., Ablasser, A., Charrel-Dennis, M., Bauernfeind, F., Horvath, G., Caffrey, D. R., et al. (2009). AIM2 recognizes cytosolic dsDNA and forms a caspase-1-activating inflammasome with ASC. Nature 458, 514-518. doi: 10.1038/nature07725

Hou, J., Zhao, R., Xia, W., Chang, C. W., You, Y., Hsu, J. M., et al. (2020). PD-L1-mediated gasdermin C expression switches apoptosis to pyroptosis in cancer cells and facilitates tumour necrosis. Nat. Cell Biol. 22, 1264-1275. doi: 10.1038/s41556-020-0575-z

Hu, Y., Jin, S., Cheng, L., Liu, G., and Jiang, Q. (2017). Autoimmune disease variants regulate GSDMB gene expression in human immune cells and whole blood. Proc. Natl. Acad. Sci. U.S.A. 114, E7860-E7862.

Humphries, F., Shmuel-Galia, L., Ketelut-Carneiro, N., Li, S., Wang, B., Nemmara, V. V., et al. (2020). Succination inactivates gasdermin D and blocks pyroptosis. Science 369, 1633-1637.

Ibrahim, J., Op de Beeck, K., Fransen, E., Croes, L., Beyens, M., et al. (2019a). Methylation analysis of Gasdermin E shows great promise as a biomarker for colorectal cancer. Cancer Med. 8, 2133-2145. doi: 10.1002/cam4.2103

Ibrahim, J., Op de Beeck, K., Fransen, E., Peeters, M., and Van Camp, G. (2019b). The Gasdermin E gene has potential as a pan-cancer biomarker, while discriminating between different tumor types. Cancers 11:1810. doi: 10.3390/ cancers 11111810

Johnson, D. C., Taabazuing, C. Y., Okondo, M. C., Chui, A. J., Rao, S. D., Brown, F. C., et al. (2018). DPP8/DPP9 inhibitor-induced pyroptosis for treatment of acute myeloid leukemia. Nat. Med. 24, 1151-1156. doi: 10.1038/s41591-0180082-y

Jorgensen, I., Zhang, Y., Krantz, B. A., and Miao, E. A. (2016). Pyroptosis triggers pore-induced intracellular traps (PITs) that capture bacteria and lead to their clearance by efferocytosis. J. Exp. Med. 213, 2113-2128. doi: 10.1084/jem. 20151613

Kambara, H., Liu, F., Zhang, X., Liu, P., Bajrami, B., Teng, Y., et al. (2018). Gasdermin D exerts anti-inflammatory effects by promoting neutrophil death. Cell Rep. 22, 2924-2936. doi: 10.1016/j.celrep.2018.02.067 
Kang, R., Zeng, L., Zhu, S., Xie, Y., Liu, J., Wen, Q., et al. (2018). Lipid peroxidation drives gasdermin D-mediated pyroptosis in lethal polymicrobial sepsis. Cell Host Microbe 24, 97-108.e104.

Karmakar, M., Katsnelson, M., Malak, H. A., Greene, N. G., Howell, S. J., Hise, A. G., et al. (2015). Neutrophil IL-1beta processing induced by pneumolysin is mediated by the NLRP3/ASC inflammasome and caspase- 1 activation and is dependent on K+ efflux. J. Immunol. 194, 1763-1775. doi: 10.4049/jimmunol. 1401624

Katoh, M., and Katoh, M. (2004a). Evolutionary recombination hotspot around GSDML-GSDM locus is closely linked to the oncogenomic recombination hotspot around the PPP1R1B-ERBB2-GRB7 amplicon. Int. J. Oncol. 24, 757763.

Katoh, M., and Katoh, M. (2004b). Identification and characterization of human DFNA5L, mouse Dfna5l, and rat Dfna5l genes in silico. Int. J. Oncol. 25, 765-770.

Kayagaki, N., Stowe, I. B., Lee, B. L., O’Rourke, K., Anderson, K., Warming, S., et al. (2015). Caspase-11 cleaves gasdermin D for non-canonical inflammasome signalling. Nature 526, 666-671. doi: 10.1038/nature15541

Kayagaki, N., Warming, S., Lamkanfi, M., Vande Walle, L., Louie, S., Dong, J., et al. (2011). Non-canonical inflammasome activation targets caspase-11. Nature $479,117-121$

Kim, M. S., Chang, X., Yamashita, K., Nagpal, J. K., Baek, J. H., Wu, G., et al. (2008). Aberrant promoter methylation and tumor suppressive activity of the DFNA5 gene in colorectal carcinoma. Oncogene 27, 3624-3634. doi: 10.1038/ sj.onc. 1211021

Kim, S., Bauernfeind, F., Ablasser, A., Hartmann, G., Fitzgerald, K. A., Latz, E., et al. (2010). Listeria monocytogenes is sensed by the NLRP3 and AIM2 inflammasome. Eur. J. Immunol. 40, 1545-1551. doi: 10.1002/eji.201040425

Knodler, L. A., Crowley, S. M., Sham, H. P., Yang, H., Wrande, M., Ma, C., et al. (2014). Noncanonical inflammasome activation of caspase-4/caspase11 mediates epithelial defenses against enteric bacterial pathogens. Cell Host Microbe 16, 249-256. doi: 10.1016/j.chom.2014.07.002

Kovarova, M., Hesker, P. R., Jania, L., Nguyen, M., Snouwaert, J. N., Xiang, Z., et al. (2012). NLRP1-dependent pyroptosis leads to acute lung injury and morbidity in mice. J. Immunol. 189, 2006-2016. doi: 10.4049/jimmunol.1201065

Kuang, S., Zheng, J., Yang, H., Li, S., Duan, S., Shen, Y., et al. (2017). Structure insight of GSDMD reveals the basis of GSDMD autoinhibition in cell pyroptosis. Proc. Natl. Acad. Sci. U.S.A. 114, 10642-10647. doi: 10.1073/pnas. 1708194114

Kusumaningrum, N., Lee, D. H., Yoon, H. S., Kim, Y. K., Park, C. H., and Chung, J. H. (2018). Gasdermin C is induced by ultraviolet light and contributes to MMP-1 expression via activation of ERK and JNK pathways. J. Dermatol. Sci. 90, 180-189. doi: 10.1016/j.jdermsci.2018.01.015

Lage, H., Helmbach, H., Grottke, C., Dietel, M., and Schadendorf, D. (2001). DFNA5 (ICERE-1) contributes to acquired etoposide resistance in melanoma cells. FEBS Lett. 494, 54-59. doi: 10.1016/s0014-5793(01)02304-3

Lei, M., Bai, X., Yang, T., Lai, X., Qiu, W., Yang, L., et al. (2012). Gsdma3 is a new factor needed for TNF-alpha-mediated apoptosis signal pathway in mouse skin keratinocytes. Histochem. Cell Biol. 138, 385-396. doi: 10.1007/s00418-0120960-1

Li, Y., Fu, T. M., Lu, A., Witt, K., Ruan, J., Shen, C., et al. (2018). Cryo-EM structures of ASC and NLRC4 CARD filaments reveal a unified mechanism of nucleation and activation of caspase-1. Proc. Natl. Acad. Sci. U.S.A. 115, 10845-10852. doi: 10.1073/pnas.1810524115

Lin, P. H., Lin, H. Y., Kuo, C. C., and Yang, L. T. (2015). N-terminal functional domain of Gasdermin A3 regulates mitochondrial homeostasis via mitochondrial targeting. J. Biomed. Sci. 22:44.

Liu, W., Kinnefors, A., Bostrom, M., Edin, F., and Rask-Andersen, H. (2013). Distribution of pejvakin in human spiral ganglion: an immunohistochemical study. Cochlear Implants Int. 14, 225-231. doi: 10.1179/1754762812y. 0000000027

Liu, X., Zhang, Z., Ruan, J., Pan, Y., Magupalli, V. G., Wu, H., et al. (2016). Inflammasome-activated gasdermin $\mathrm{D}$ causes pyroptosis by forming membrane pores. Nature 535, 153-158. doi: 10.1038/nature18629

Liu, Z., Wang, C., Yang, J., Chen, Y., Zhou, B., Abbott, D. W., et al. (2020). Caspase1 engages full-length gasdermin $\mathrm{D}$ through two distinct interfaces that mediate caspase recruitment and substrate cleavage. Immunity 53, 106-114.e105.
Lu, A., Li, Y., Yin, Q., Ruan, J., Yu, X., Egelman, E., et al. (2015). Plasticity in PYD assembly revealed by cryo-EM structure of the PYD filament of AIM2. Cell Discov. 1:15013.

Lutkowska, A., Roszak, A., Lianeri, M., Sowinska, A., Sotiri, E., and Jagodzinski, P. P. (2017). Analysis of rs8067378 Polymorphism in the risk of uterine cervical cancer from a polish population and its impact on gasdermin B expression. Mol. Diagn. Ther. 21, 199-207. doi: 10.1007/s40291-017-0256-1

Man, S. M., Ekpenyong, A., Tourlomousis, P., Achouri, S., Cammarota, E., Hughes, K., et al. (2014). Actin polymerization as a key innate immune effector mechanism to control Salmonella infection. Proc. Natl. Acad. Sci. U.S.A. 111, 17588-17593. doi: 10.1073/pnas.1419925111

Mariathasan, S., Weiss, D. S., Newton, K., McBride, J., O’Rourke, K., Roose-Girma, M., et al. (2006). Cryopyrin activates the inflammasome in response to toxins and ATP. Nature 440, 228-232. doi: 10.1038/nature04515

Miao, E. A., Alpuche-Aranda, C. M., Dors, M., Clark, A. E., Bader, M. W., Miller, S. I., et al. (2006). Cytoplasmic flagellin activates caspase-1 and secretion of interleukin lbeta via Ipaf. Nat. Immunol. 7, 569-575. doi: 10.1038/ ni1344

Miao, E. A., Leaf, I. A., Treuting, P. M., Mao, D. P., Dors, M., Sarkar, A., et al. (2010a). Caspase-1-induced pyroptosis is an innate immune effector mechanism against intracellular bacteria. Nat. Immunol. 11, 1136-1142. doi: 10.1038/ni.1960

Miao, E. A., Mao, D. P., Yudkovsky, N., Bonneau, R., Lorang, C. G., Warren, S. E., et al. (2010b). Innate immune detection of the type III secretion apparatus through the NLRC4 inflammasome. Proc. Natl. Acad. Sci. U.S.A. 107, 30763080. doi: $10.1073 /$ pnas. 0913087107

Miguchi, M., Hinoi, T., Shimomura, M., Adachi, T., Saito, Y., Niitsu, H., et al. (2016). Gasdermin C is upregulated by inactivation of transforming growth factor beta receptor type II in the presence of mutated APC, promoting colorectal cancer proliferation. PLoS One 11:e0166422. doi: 10.1371/journal. pone. 0166422

Misawa, T., Takahama, M., Kozaki, T., Lee, H., Zou, J., Saitoh, T., et al. (2013). Microtubule-driven spatial arrangement of mitochondria promotes activation of the NLRP3 inflammasome. Nat. Immunol. 14, 454-460. doi: 10.1038/ni. 2550

Moghaddas, F., Zeng, P., Zhang, Y., Schutzle, H., Brenner, S., Hofmann, S. R., et al. (2018). Autoinflammatory mutation in NLRC4 reveals a leucine-rich repeat (LRR)-LRR oligomerization interface. J. Allergy Clin. Immunol. 142, 1956-1967.e1956.

Molina-Crespo, A., Cadete, A., Sarrio, D., Gamez-Chiachio, M., Martinez, L., Chao, K., et al. (2019). Intracellular delivery of an antibody targeting gasdermin-B reduces HER2 breast cancer aggressiveness. Clin. Cancer Res. 25, 4846-4858. doi: 10.1158/1078-0432.ccr-18-2381

Mujtaba, G., Bukhari, I., Fatima, A., and Naz, S. (2012). A p.C343S missense mutation in PJVK causes progressive hearing loss. Gene 504, 98-101. doi: 10.1016/j.gene.2012.05.013

Mulvihill, E., Sborgi, L., Mari, S. A., Pfreundschuh, M., Hiller, S., and Muller, D. J. (2018). Mechanism of membrane pore formation by human gasdermin-D. EMBO J. 37:e98321.

Newman, Z. L., Leppla, S. H., and Moayeri, M. (2009). CA-074Me protection against anthrax lethal toxin. Infect. Immun. 77, 4327-4336. doi: 10.1128/iai. 00730-09

Orning, P., Weng, D., Starheim, K., Ratner, D., Best, Z., Lee, B., et al. (2018). Pathogen blockade of TAK1 triggers caspase-8-dependent cleavage of gasdermin D and cell death. Science 362, 1064-1069. doi: 10.1126/science. aau2818

Panganiban, R. A., Sun, M., Dahlin, A., Park, H. R., Kan, M., Himes, B. E., et al. (2018). A functional splice variant associated with decreased asthma risk abolishes the ability of gasdermin B to induce epithelial cell pyroptosis. J. Allergy Clin. Immunol. 142, 1469-1478.e1462.

Pardo, J., Wallich, R., Martin, P., Urban, C., Rongvaux, A., Flavell, R. A., et al. (2008). Granzyme B-induced cell death exerted by ex vivo CTL: discriminating requirements for cell death and some of its signs. Cell Death. Differ. 15, 567-579. doi: 10.1038/sj.cdd.4402289

Pawlus, M. R., Wang, L., and Hu, C. J. (2014). STAT3 and HIF1alpha cooperatively activate HIF1 target genes in MDA-MB-231 and RCC4 cells. Oncogene 33, 1670-1679. doi: 10.1038/onc.2013.115 
Poltorak, A., He, X., Smirnova, I., Liu, M. Y., Van Huffel, C., Du, X., et al. (1998). Defective LPS signaling in $\mathrm{C} 3 \mathrm{H} / \mathrm{HeJ}$ and $\mathrm{C} 57 \mathrm{BL} / 10 \mathrm{ScCr}$ mice: mutations in Tlr4 gene. Science 282, 2085-2088. doi: 10.1126/science.282.5396.2085

Proell, M., Gerlic, M., Mace, P. D., Reed, J. C., and Riedl, S. J. (2013). The CARD plays a critical role in ASC foci formation and inflammasome signalling. Biochem. J. 449, 613-621. doi: 10.1042/bj20121198

Qiu, R., Zhang, H., Zhao, H., Li, J., Guo, C., Gong, Y., et al. (2013). Genetic variants on $17 \mathrm{q} 21$ are associated with ankylosing spondylitis susceptibility and severity in a Chinese Han population. Scand. J. Rheumatol. 42, 469-472. doi: 10.3109/03009742.2013.786755

Rathinam, V. A., Jiang, Z., Waggoner, S. N., Sharma, S., Cole, L. E., Waggoner, L., et al. (2010). The AIM2 inflammasome is essential for host defense against cytosolic bacteria and DNA viruses. Nat. Immunol. 11, 395-402. doi: 10.1038/ ni. 1864

Rogers, C., Erkes, D. A., Nardone, A., Aplin, A. E., Fernandes-Alnemri, T., and Alnemri, E. S. (2019). Gasdermin pores permeabilize mitochondria to augment caspase- 3 activation during apoptosis and inflammasome activation. Nat. Commun. 10:1689.

Rogers, C., Fernandes-Alnemri, T., Mayes, L., Alnemri, D., Cingolani, G., and Alnemri, E. S. (2017). Cleavage of DFNA5 by caspase-3 during apoptosis mediates progression to secondary necrotic/pyroptotic cell death. Nat. Commun. 8:14128.

Ruhl, S., and Broz, P. (2015). Caspase-11 activates a canonical NLRP3 inflammasome by promoting $\mathrm{K}(+)$ efflux. Eur. J. Immunol. 45, 2927-2936. doi: 10.1002/eji.201545772

Ruhl, S., Shkarina, K., Demarco, B., Heilig, R., Santos, J. C., and Broz, P. (2018). ESCRT-dependent membrane repair negatively regulates pyroptosis downstream of GSDMD activation. Science 362, 956-960. doi: 10.1126/science. aar7607

Saeki, N., Kim, D. H., Usui, T., Aoyagi, K., Tatsuta, T., Aoki, K., et al. (2007). GASDERMIN, suppressed frequently in gastric cancer, is a target of LMO1 in TGF-beta-dependent apoptotic signalling. Oncogene 26, 6488-6498. doi: 10.1038/sj.onc. 1210475

Saeki, N., Kuwahara, Y., Sasaki, H., Satoh, H., and Shiroishi, T. (2000). Gasdermin (Gsdm) localizing to mouse Chromosome 11 is predominantly expressed in upper gastrointestinal tract but significantly suppressed in human gastric cancer cells. Mamm. Genome 11, 718-724. doi: 10.1007/s003350010138

Saeki, N., Usui, T., Aoyagi, K., Kim, D. H., Sato, M., Mabuchi, T., et al. (2009). Distinctive expression and function of four GSDM family genes (GSDMA-D) in normal and malignant upper gastrointestinal epithelium. Genes Chromosomes Cancer 48, 261-271. doi: 10.1002/gcc.20636

Sarhan, J., Liu, B. C., Muendlein, H. I., Li, P., Nilson, R., Tang, A. Y., et al. (2018). Caspase- 8 induces cleavage of gasdermin D to elicit pyroptosis during Yersinia infection. Proc. Natl. Acad. Sci. U.S.A. 115, E10888-E10897.

Sborgi, L., Ruhl, S., Mulvihill, E., Pipercevic, J., Heilig, R., Stahlberg, H., et al. (2016). GSDMD membrane pore formation constitutes the mechanism of pyroptotic cell death. EMBO J. 35, 1766-1778. doi: 10.15252/embj.20169 4696

Shen, X., Zhang, Q., He, Z., Xiao, S., Li, H., and Huang, Z. (2020). Overexpression of gasdermin D promotes invasion of adenoid cystic carcinoma. Int. J. Clin. Exp. Pathol. 13, 1802-1811.

Shi, J., Zhao, Y., Wang, K., Shi, X., Wang, Y., Huang, H., et al. (2015). Cleavage of GSDMD by inflammatory caspases determines pyroptotic cell death. Nature 526, 660-665. doi: 10.1038/nature 15514

Shi, J., Zhao, Y., Wang, Y., Gao, W., Ding, J., Li, P., et al. (2014). Inflammatory caspases are innate immune receptors for intracellular LPS. Nature 514, 187192. doi: $10.1038 /$ nature 13683

Shi, P., Tang, A., Xian, L., Hou, S., Zou, D., Lv, Y., et al. (2015). Loss of conserved Gsdma3 self-regulation causes autophagy and cell death. Biochem. J. 468, 325-336. doi: 10.1042/bj20150204

Soderman, J., Berglind, L., and Almer, S. (2015). Gene expression-genotype analysis implicates GSDMA, GSDMB, and LRRC3C as contributors to inflammatory bowel disease susceptibility. Biomed. Res. Int. 2015:834805.

Stutz, A., Kolbe, C. C., Stahl, R., Horvath, G. L., Franklin, B. S., van Ray, O., et al. (2017). NLRP3 inflammasome assembly is regulated by phosphorylation of the pyrin domain. J. Exp. Med. 214, 1725-1736. doi: 10.1084/jem.20160933

Tamura, M., Tanaka, S., Fujii, T., Aoki, A., Komiyama, H., Ezawa, K., et al. (2007). Members of a novel gene family, Gsdm, are expressed exclusively in the epithelium of the skin and gastrointestinal tract in a highly tissue-specific manner. Genomics 89, 618-629. doi: 10.1016/j.ygeno.2007.01.003

Tanaka, S., Mizushina, Y., Kato, Y., Tamura, M., and Shiroishi, T. (2013). Functional conservation of Gsdma cluster genes specifically duplicated in the mouse genome. G3 Bethesda 3, 1843-1850. doi: 10.1534/g3.113.007393

Tang, Z., Ji, L., Han, M., Xie, J., Zhong, F., Zhang, X., et al. (2020). Pyroptosis is involved in the inhibitory effect of FL118 on growth and metastasis in colorectal cancer. Life Sci. 257:118065. doi: 10.1016/j.lfs.2020.118065

Terao, C., Kawaguchi, T., Dieude, P., Varga, J., Kuwana, M., Hudson, M., et al. (2017). Transethnic meta-analysis identifies GSDMA and PRDM1 as susceptibility genes to systemic sclerosis. Ann. Rheum. Dis. 76, 1150-1158. doi: 10.1136/annrheumdis-2016-210645

Thomas, P. G., Dash, P., Aldridge, J. R. Jr., Ellebedy, A. H., Reynolds, C., et al. (2009). The intracellular sensor NLRP3 mediates key innate and healing responses to influenza a virus via the regulation of caspase-1. Immunity 30, 566-575. doi: 10.1016/j.immuni.2009.02.006

Ting, J. P., Lovering, R. C., Alnemri, E. S., Bertin, J., Boss, J. M., Davis, B. K., et al. (2008). The NLR gene family: a standard nomenclature. Immunity 28, 285-287. doi: 10.1016/j.immuni.2008.02.005

Van Laer, L., Huizing, E. H., Verstreken, M., van Zuijlen, D., Wauters, J. G., Bossuyt, P. J., et al. (1998). Nonsyndromic hearing impairment is associated with a mutation in DFNA5. Nat. Genet. 20, 194-197. doi: 10.1038/2503

Van Laer, L., Van Camp, G., van Zuijlen, D., Green, E. D., Verstreken, M., Schatteman, I., et al. (1997). Refined mapping of a gene for autosomal dominant progressive sensorineural hearing loss (DFNA5) to a 2-cM region, and exclusion of a candidate gene that is expressed in the cochlea. Eur. J. Hum. Genet. 5, 397-405. doi: 10.1159/000484798

Wang, C. J., Tang, L., Shen, D. W., Wang, C., Yuan, Q. Y., Gao, W., et al. (2013). The expression and regulation of DFNA5 in human hepatocellular carcinoma DFNA5 in hepatocellular carcinoma. Mol. Biol. Rep. 40, 6525-6531. doi: 10. 1007/s11033-013-2581-8

Wang, K., Sun, Q., Zhong, X., Zeng, M., Zeng, H., Shi, X., et al. (2020a). Structural mechanism for GSDMD targeting by autoprocessed caspases in pyroptosis. Cell 180, 941-955.e920.

Wang, W. J., Chen, D., Jiang, M. Z., Xu, B., Li, X. W., Chu, Y., et al. (2018a). Downregulation of gasdermin $\mathrm{D}$ promotes gastric cancer proliferation by regulating cell cycle-related proteins. J. Dig. Dis. 19, 74-83. doi: 10.1111/17512980.12576

Wang, X., Li, X., Gong, P., Zhang, N., Li, L., Ouyang, H., et al. (2020b). Pyroptosis executioner gasdermin D contributes to host defense and promotes Th 1 immune response during Neospora caninum infection. Vet. Parasitol. 286:109254. doi: 10.1016/j.vetpar.2020.109254

Wang, Y., Gao, W., Shi, X., Ding, J., Liu, W., He, H., et al. (2017). Chemotherapy drugs induce pyroptosis through caspase-3 cleavage of a gasdermin. Nature 547, 99-103. doi: 10.1038/nature22393

Wang, Y., Yin, B., Li, D., Wang, G., Han, X., and Sun, X. (2018b). GSDME mediates caspase-3-dependent pyroptosis in gastric cancer. Biochem. Biophys. Res. Commun. 495, 1418-1425. doi: 10.1016/j.bbrc.2017.11.156

Watabe, K., Ito, A., Asada, H., Endo, Y., Kobayashi, T., Nakamoto, K., et al. (2001). Structure, expression and chromosome mapping of MLZE, a novel gene which is preferentially expressed in metastatic melanoma cells. Jpn. J. Cancer Res. 92, 140-151. doi: 10.1111/j.1349-7006.2001.tb01076.x

Webb, M. S., Miller, A. L., and Thompson, E. B. (2007). In CEM cells the autosomal deafness gene dfna5 is regulated by glucocorticoids and forskolin. J. Steroid. Biochem. Mol. Biol. 107, 15-21. doi: 10.1016/j.jsbmb.2007.02.004

Wu, C., Lu, W., Zhang, Y., Zhang, G., Shi, X., Hisada, Y., et al. (2019). Inflammasome activation triggers blood clotting and host death through pyroptosis. Immunity 50, 1401-1411.e1404.

Wu, J., Fernandes-Alnemri, T., and Alnemri, E. S. (2010). Involvement of the AIM2, NLRC4, and NLRP3 inflammasomes in caspase-1 activation by Listeria monocytogenes. J. Clin. Immunol. 30, 693-702. doi: 10.1007/s10875-0109425-2

Wu, X., Mao, X., Huang, Y., Zhu, Q., Guan, J., and Wu, L. (2020). Detection of proteins associated with the pyroptosis signaling pathway in breast cancer tissues and their significance. Int. J. Clin. Exp. Pathol. 13, 1408-1414.

Xi, G., Gao, J., Wan, B., Zhan, P., Xu, W., Lv, T., et al. (2019). GSDMD is required for effector CD8(+) $\mathrm{T}$ cell responses to lung cancer cells. Int. Immunopharmacol. 74:105713. doi: 10.1016/j.intimp.2019.105713 
Xia, S., Ruan, J., and Wu, H. (2019). Monitoring gasdermin pore formation in vitro. Methods Enzymol. 625, 95-107. doi: 10.1016/bs.mie.2019.04.024

Xu, B., Jiang, M., Chu, Y., Wang, W., Chen, D., Li, X., et al. (2018). Gasdermin D plays a key role as a pyroptosis executor of non-alcoholic steatohepatitis in humans and mice. J. Hepatol. 68, 773-782. doi: 10.1016/j.jhep.2017.11.040

Xu, H., Yang, J., Gao, W., Li, L., Li, P., Zhang, L., et al. (2014). Innate immune sensing of bacterial modifications of Rho GTPases by the Pyrin inflammasome. Nature 513, 237-241. doi: 10.1038/nature13449

Yang, H., Hreggvidsdottir, H. S., Palmblad, K., Wang, H., Ochani, M., Li, J., et al. (2010). A critical cysteine is required for HMGB1 binding to Toll-like receptor 4 and activation of macrophage cytokine release. Proc. Natl. Acad. Sci. U.S.A. 107, 11942-11947. doi: 10.1073/pnas.1003893107

Yang, J., Liu, Z., Wang, C., Yang, R., Rathkey, J. K., Pinkard, O. W., et al. (2018). Mechanism of gasdermin D recognition by inflammatory caspases and their inhibition by a gasdermin D-derived peptide inhibitor. Proc. Natl. Acad. Sci. U.S.A. 115, 6792-6797. doi: 10.1073/pnas. 1800562115

Yu, J. W., Fernandes-Alnemri, T., Datta, P., Wu, J., Juliana, C., Solorzano, L., et al. (2007). Pyrin activates the ASC pyroptosome in response to engagement by autoinflammatory PSTPIP1 mutants. Mol. Cell 28, 214-227. doi: 10.1016/j. molcel.2007.08.029

Yu, J., Kang, M. J., Kim, B. J., Kwon, J. W., Song, Y. H., Choi, W. A., et al. (2011). Polymorphisms in GSDMA and GSDMB are associated with asthma susceptibility, atopy and BHR. Pediatr. Pulmonol. 46, 701-708. doi: 10.1002/ ppul.21424

Yu, J., Li, S., Qi, J., Chen, Z., Wu, Y., Guo, J., et al. (2019). Cleavage of GSDME by caspase-3 determines lobaplatin-induced pyroptosis in colon cancer cells. Cell Death Dis. 10:193.
Zhang, C. C., Li, C. G., Wang, Y. F., Xu, L. H., He, X. H., Zeng, Q. Z., et al. (2019). Chemotherapeutic paclitaxel and cisplatin differentially induce pyroptosis in A549 lung cancer cells via caspase-3/GSDME activation. Apoptosis 24, 312-325. doi: 10.1007/s10495-019-01515-1

Zhang, Z., Zhang, Y., Xia, S., Kong, Q., Li, S., Liu, X., et al. (2020). Gasdermin E suppresses tumour growth by activating anti-tumour immunity. Nature 579, 415-420. doi: 10.1038/s41586-020-2071-9

Zhou, Y., Jiang, X., Gu, P., Chen, W., Zeng, X., and Gao, X. (2012). Gsdma3 mutation causes bulge stem cell depletion and alopecia mediated by skin inflammation. Am. J. Pathol. 180, 763-774. doi: 10.1016/j.ajpath.2011.10.034

Zhou, Z., He, H., Wang, K., Shi, X., Wang, Y., Su, Y., et al. (2020). Granzyme a from cytotoxic lymphocytes cleaves GSDMB to trigger pyroptosis in target cells. Science 368:eaaz7548. doi: 10.1126/science.aaz7548

Zychlinsky, A., Prevost, M. C., and Sansonetti, P. J. (1992). Shigella flexneri induces apoptosis in infected macrophages. Nature 358, 167-169. doi: 10.1038/ $358167 \mathrm{a} 0$

Conflict of Interest: The authors declare that the research was conducted in the absence of any commercial or financial relationships that could be construed as a potential conflict of interest.

Copyright $\odot 2021$ Wang, Chen and Zhang. This is an open-access article distributed under the terms of the Creative Commons Attribution License (CC BY). The use, distribution or reproduction in other forums is permitted, provided the original author(s) and the copyright owner(s) are credited and that the original publication in this journal is cited, in accordance with accepted academic practice. No use, distribution or reproduction is permitted which does not comply with these terms. 